\title{
Development of TOPSIS Technique under Pythagorean Fuzzy Hypersoft Environment Based on Correlation Coefficient and Its Application towards the Selection of Antivirus Mask in COVID-19 Pandemic
}

\author{
Rana Muhammad Zulqarnain $\left(\mathbb{D},{ }^{1}\right.$ Imran Siddique, ${ }^{2}$ Fahd Jarad $\mathbb{D}^{3,4}$ Rifaqat Ali, ${ }^{5}$ \\ and Thabet Abdeljawad $\mathbb{D}^{6,7}$ \\ ${ }^{1}$ Department of Mathematics, University of Management and Technology Lahore, Sialkot Campus, Pakistan \\ ${ }^{2}$ Department of Mathematics, School of Science, University of Management and Technology, Lahore, Pakistan \\ ${ }^{3}$ Department of Mathematics, Cankaya University, Etimesgut, Ankara, Turkey \\ ${ }^{4}$ Department of Medical Research, China Medical University Hospital, China Medical University, Taichung, Taiwan \\ ${ }^{5}$ Department of Mathematics, College of Science and Arts, King Khalid University, Muhayil, 61413 Abha, Saudi Arabia \\ ${ }^{6}$ Department of Mathematics and General Sciences, Prince Sultan University, Riyadh, Saudi Arabia \\ ${ }^{7}$ Department of Computer Science and Information Engineering, Asia University, Taichung, Taiwan
}

Correspondence should be addressed to Fahd Jarad; fahd@cankaya.edu.tr and Thabet Abdeljawad; tabdeljawad@psu.edu.sa

Received 29 December 2020; Revised 24 January 2021; Accepted 9 February 2021; Published 17 March 2021

Academic Editor: Ahmed Mostafa Khalil

Copyright @ 2021 Rana Muhammad Zulqarnain et al. This is an open access article distributed under the Creative Commons Attribution License, which permits unrestricted use, distribution, and reproduction in any medium, provided the original work is properly cited.

\begin{abstract}
The correlation coefficient between two variables plays an important role in statistics. Also, the accuracy of relevance assessment depends on information from a set of discourses. The data collected from numerous statistical studies are full of exceptions. The Pythagorean fuzzy hypersoft set (PFHSS) is a parameterized family that deals with the subattributes of the parameters and an appropriate extension of the Pythagorean fuzzy soft set. It is also the generalization of the intuitionistic fuzzy hypersoft set (IFHSS), which is used to accurately assess insufficiency, anxiety, and uncertainties in decision-making. The PFHSS can accommodate more uncertainties compared to the IFHSS, and it is the most substantial methodology to describe fuzzy information in the decision-making process. The core objective of the this study is to develop the notion and features of the correlation coefficient and the weighted correlation coefficient for PFHSS and to introduce the aggregation operators such as Pythagorean fuzzy hypersoft weighted average (PFHSWA) and Pythagorean fuzzy hypersoft weighted geometric (PFHSWG) operators under the PFHSS scenario. A prioritization technique for order preference by similarity to the ideal solution (TOPSIS) under PFHSS based on correlation coefficients and weighted correlation coefficients is presented. Through the developed methodology, a technique for solving multiattribute group decision-making (MAGDM) problem is planned. Also, the importance of the developed methodology and its application in indicating multipurpose antivirus mask throughout the COVID-19 pandemic period is presented. A brief comparative analysis is described with the advantages, effectiveness, and flexibility of numerous existing studies that demonstrate the effectiveness of the proposed method.
\end{abstract}

\section{Introduction}

Decision-making (DM) is one of the most interesting issues these days to choose an appropriate alternative for any particular purpose. Initially, it is assumed that information regarding possible choices is collected in crisp numbers, but in real-life scenarios, collective facts and figures always comprises incorrect and imprecise information. Correlation plays the main role in statistics as well as engineering. Through correlation analysis, the joint relationship of two variables can be found and used to evaluate the interdependence of two variables. Other than this, 
probabilistic strategies have been applied to so many practical engineering issues, but there are many limitations. For example, the probability of the process is dependent on a lot of information, which may be haphazard. However, large complex structures have numerous incomprehensible uncertainties, and it is usually difficult to obtain accurate probability events. Therefore, due to incomprehensive quantitative information, consequences beneath probability theory do not provide useful information for experts. Besides, in practical applications, there are no adequate data to correctly process renowned statistical data. Due to the abovementioned barriers, consequences based on probability theory are not obtainable to experts at all time. So, probabilistic methods are usually insufficient to resolve specified procured uncertainties in the data. Quite a lot of investigators in the world have projected and advised different methods to solve such difficulties that contain vagueness. Zadeh proposed the idea of fuzzy sets (FSs) [1] to solve complex problems that contain uncertainness as well as ambiguity. In some cases, we must check membership as a nonmembership value in the representation of objects that cannot be handled by FS. To overcome this concern, Atanassov proposed the concept of intuitionistic fuzzy sets (IFSs) [2]. Based on this, theories, such as cubic IFS [3], interval-valued IFS [4], and linguistic interval-valued IFS [5], have been developed and used by investigators.

Ultimately, based on the above theories, experts observed that the sum of their membership and non-membership cannot exceed one. Atanassov's IFS only deals with insufficient data due to membership and nonmembership values, while IFS cannot deal with inappropriate and vague information. Thus, the above work is used to visualize our surroundings of linear inequality between the degree of membership (MD) and the degree of nonmembership (NMD). However, if the decision-maker goes steady with object $\mathrm{MD}=0.8$ and $\mathrm{NDM}=0.5$, then $0.8+0.5 \not 1$. Clearly, we can see that it cannot be handled by the abovementioned IFS theories. To overcome the abovementioned limitations, Yager [6, 7] extended the IFS to Pythagorean fuzzy sets (PFSs) by modifying the condition $\mathscr{T}+\mathscr{J} \leq 1$ to $\mathscr{T}^{2}+\mathscr{J}^{2} \leq 1$. Zhang and Xu [8] defined some operational laws and extended the TOPSIS technique to solve MCDM problems under PFS environment. Wei and Lu [9] presented several Pythagorean fuzzy power aggregation operators with their properties and proposed the decision-making approaches to solve MADM problems based on developed operators. Wang and $\mathrm{Li}$ [10] proposed the Pythagorean fuzzy interaction operational laws and power Bonferroni mean operators. Then, they discussed some specific cases of established operators and considered their properties.

Zhang [11] established a novel decision-making technique based on Pythagorean fuzzy numbers (PFNs) to solve multiple criteria group decision-making (MCGDM) problems. He also developed the accuracy function for the ranking of PFNs and similarity measures under a PFS environment with some desirable properties. Garg [12] extended the weighted aggregation operators to PFSs and developed several operators and presented a decisionmaking approach based on developed operators. Peng and
Yang [13] proposed division and subtraction operators, investigated their properties based on PFSs, and developed a ranking method based on developed operators to solve MAGDM problems. Garg [14] introduced the logarithmic operational laws with several weighted averaging and weighted geometric operators based on PFSs. Gao et al. [15] established several Pythagorean fuzzy interaction operators by using arithmetic and geometric operations and proposed some decision-making approaches to solve MADM problems. Peng and Yuan [16] presented the Pythagorean fuzzy point operators and established decision-making approaches to solve MADM problems based on developed operators. Ma and Xu [17] improved the score and accuracy functions for PFNs and developed novel averaging and geometric operators based on PFS information.

All the above approaches are widely applied in many areas and fields. However, these theories have limitations due to their incompetence with the parameterization tool. To overcome this kind of complexity, Molodtsov [18] proposed a general mathematical parameterization tool soft set (SS), which is used to deal with uncertain, ambiguous, and indeterminate components, in which certain specific parameters of the object are evaluated. Maji et al. [19] extended the concept of SS, proposed some operations with their several properties, and used the established concepts for decision-making [20]. Maji et al. [21] planned the idea of fuzzy soft sets (FSSs) by combining FSs and SSs. In addition, they projected an intuitionistic fuzzy soft set (IFSS) with fundamental operations along with properties [22]. Garg and Arora [23] extended the generalized version of the IFSS with weighted averaging and geometric aggregation operators and built a decisionmaking technique to resolve complications beneath an intuitionistic fuzzy environment. Garg [24] developed some improved score functions to analyze the ranking of the normal intuitionistic and interval-valued intuitionistic sets and established new methodologies to solve multiattribute decision-making (MADM) problems. The idea of entropy measure and TOPSIS based on correlation coefficient (CC) has been developed by using complex qrung orthopair fuzzy information and used the established strategies for decision-making [25]. Garg and Arora [26] developed aggregate operators by using dual hesitant fuzzy soft numbers and utilized the proposed operators to solve multicriteria decision-making (MCDM) problems. To measure the relationship among dual hesitant fuzzy soft set, Arora and Garg [27] introduced the CC and developed a decision-making approach under the presented environment to solve the MCDM approach, and they also used the proposed methodology for decisionmaking, medical diagnoses, and pattern recognition. They also developed operational laws and presented some prioritized aggregation operators under linguistic IFS environment [28] and extended the Maclaurin symmetric mean (MSM) operators to IFSSs based on Archimedean Tconorm and T-norm [29]. Garg and Arora introduced the correlation measures on IFSSs and constructed the TOPSIS technique on developed correlation measures [30]. 
In this era, the assumptions and application scenarios of SSs and the abovementioned different research extensions are developing rapidly. Peng et al. competently solved the abovementioned complications [31] and proposed a novel idea of Pythagorean fuzzy soft sets (PFSSs) by combining the two existing theories PFS and SS with some basic operations with ideal characteristics. Athira et al. [32] developed an entropy measure based on PFSS information. They also proposed the Hamming distance and Euclidean distance of PFSSs and used them for decision-making [33]. As far as the author knows, there are few studies on the theory of PFSSs. Therefore, it is a better way to keep PFSSs flexible than IFSSs or FSSs. Naeem et al. [34] established some operations with their desirable properties and extended the TOPSIS and VIKOR techniques to develop the affluence of linguistic variables based on PFSS information. They also rendered an application on the consequence of stock exchange investment by utilizing the developed techniques. Riaz et al. [35] established the TOPSIS technique for $m$-polar PFSSs and presented an example to solve MCGDM problems within a considered hybrid structure. They also introduced similarity measures for PFSSs [36]. Hwang and Yoon [37] developed TOPSIS to solve decision-making problems. By using the TOPSIS method, we can easily obtain the minimum distance from a positive ideal solution which supports electing the finest alternative. After the TOPSIS method was developed, many researchers used the TOPSIS method for decision-making and extended this method to fuzzy and intuitionistic fuzzy environments [38-51].

Smarandache [52] extended the concept of SS to hypersoft sets (HSSs) by replacing the one-parameter function $f$ with a multiparameter (subattribute) function described on the Cartesian product of $n$ attributes. The established HSS is more flexible than SS and more suitable for the decision-making environments. He also launched advanced extensions of HSSs, including crisp HSS, fuzzy HSS, and intuitionistic fuzzy HSS. Nowadays, HSS theory and its extensions have been rising unexpectedly. Several investigators went through progressed distinctive operators along with characteristics based on HSS and its extensions [53-55]. Zulqarnain et al. [56] extended the notion of IFHSSs and developed some aggregation operators and TOPSIS technique based on CC under the IFHSS scenario. They also established a decision-making technique by utilizing the proposed TOPSIS technique to resolve the MADM problem. Thus, the above work is considered to examine the environment of linear inequality between the degree of membership (MD) and the degree of nonmembership (NMD) of attributes and subattributes of the considered parameters. However, if the decision-maker stabilizes when the target $\mathrm{MD}=0.7$ and $\mathrm{NDM}=0.6$, then $0.7+0.6 \not 1$. Obviously, we can see that it cannot be handled by the abovementioned theories. To overcome the above limitations, we prolonged the IFHSS to PFHSS by modifying the condition $\mathscr{T}_{\mathscr{F}(\breve{d})}(\delta)+\mathscr{F}_{\mathscr{F}(\breve{d})}(\delta) \leq 1 \quad$ to $\quad\left(\mathscr{T}_{\mathscr{F}(\check{d})}(\delta)\right)^{2}+$ $\left(\mathscr{J}_{\mathscr{F}(\check{d})}(\delta)\right)^{2} \leq 1$.

It is true that everyone is eager to purchase the smartest mask for the present terrible virus, but distinct groups of people must have different essential desires. Particularly during the COVID-19 pandemic, it is our responsibility to use good and effective antivirus masks to reduce the COVID-19 effects. Thus, for different groups of people, logically picking out and using masks are of key importance. The best mask does not mean the most expensive mask. For the majority of people, it is not necessary to use identical masks like those used by frontline medical examination staff. The use of a gas mask not only depends on masks but also depends on the human face. Besides, in serious pandemic scenarios and shortage of gas masks, the choice of mask could also be associated with components like reusability along with the quality of raw materials. Thus, the majority of people, in serious pandemic scenarios, consider multiple components while choosing masks to enhance the reapportionment of medical resources. But, with the extraordinary information of the people as well as the uncertainty of the increase in COVID-19, the difficulty increases. The majority of people are unable to choose a good antivirus mask; mostly people prefer the cheapest antivirus mask. Yang et al. [57] and Shahzadi and Akram [58] used the spherical normal fuzzy sets and formation fuzzy soft Yager ordered weighted average and geometric operators for the selection of effective gas masks during the COVID-19 pandemic.

In this study, the TOPSIS technique was extended to PFHSS information, and the mechanism was based on the assumption of PFHSNs. To measure the degree of dependence on PHFSS, we proposed CC and WCC on PFHSSs and studied some properties of the developed CC. To accurately achieve the goal, the given TOPSIS technique can be extended to solve the MAGDM problem. In this research, our main goal is to introduce the CC and WCC based on the PHFSS information and develop the TOPSIS method of PFHSSs based on the proposed CC. In order to solve the MAGDM problem based on the extended TOPSIS method, an algorithm was developed, and the effectiveness of the proposed technique was verified by a numerical example. Given correlation measures for PFHSSs have been considered for paired PFHSSs, which will be used to calculate the interrelationship between elements and the scope of dependence. Since the existing IFS and IFSS, PFS, PFSS, and IFHSS are special cases of PFHSS, the measures that have been formulated are more general than the existing measures. The correlation coefficient retains the linear relationship between considered elements. To find the general closeness coefficient by the TOPSIS method, generally, the researchers used the similarity measure and distance. But, in our developed TOPSIS technique, the closeness coefficient can be calculated by using the correlation coefficient.

The rest of this article is organized as follows. In Section 2, we recollect some basic definitions, such as SS, HSS, IFHSS, and PFHSS, which will be used to construct the structure of this article. In Section 3, we proposed the informational energies and correlation measures for PFHSSs and used the established terminologies to develop CC and WCC and their properties. Some aggregation operators are also introduced such as Pythagorean fuzzy hypersoft weighted average (PFHSWA) and Pythagorean fuzzy hypersoft weighted geometric (PFHSWG) operators. An 
extended TOPSIS technique based on CC in the PFHSS environment is proposed, and an algorithm is developed based on the proposed TOPSIS method to solve the MAGDM problem, and a numerical description is given in Section 4. Also, we use some existing techniques to propose comparative studies between our proposed methods. Similarly, the advantages, naivety, flexibility, and effectiveness of planned algorithms are introduced. We have a brief discussion and comparative analysis of the proposed methods and existing techniques in Section 5.

\section{Preliminaries}

In this section, we recollect some basic definitions that help build the structure of the article such as soft set, hypersoft set, fuzzy hypersoft set, IFHSS, and PFHSS.

Definition 1 (see [18]). Let $\mathscr{U}$ be the universal set and $\mathscr{E}$ be the set of attributes concerning $\mathscr{U}$. Let $\mathscr{P}(\mathscr{U})$ be the power set of $\mathcal{U}$ and $\mathscr{A} \subseteq \mathscr{E}$. A pair $(\mathscr{F}, \mathscr{A})$ is called a soft set over $\mathcal{U}$, and its mapping is given as

$$
\mathscr{F}: \mathscr{A} \longrightarrow \mathscr{P}(\mathscr{U}) \text {. }
$$

It is also defined as

$$
(\mathscr{F}, \mathscr{A})=\{\mathscr{F}(e) \in \mathscr{P}(\mathscr{U}): e \in \epsilon, \mathscr{F}(e)=\varnothing, \quad \text { if } e \notin \mathscr{A}\} .
$$

Maji et al. [21] explored the theory of FS and SS and planned a more generalized version to handle the uncertainty compared with the existing FS and SS along with its unique features. This is generally known as a fuzzy soft set, which is a combination of FS and SS.

Definition 2 (see [21]). Let $\mathscr{F}(\mathcal{U})$ be a collection of all fuzzy subsets over $\mathscr{U}$ and $\mathscr{E}$ be a set of attributes. Let $\mathscr{A} \subseteq \mathscr{E}$, then a pair $(\mathscr{F}, \mathscr{A})$ is called FSS over $\mathscr{U}$, where $\mathscr{F}$ is a mapping such as $\mathscr{F}: \mathscr{A} \longrightarrow F(\mathcal{U})$.

Definition 3 (see [52]). Let $\mathcal{U}$ be a universe of discourse, $\mathscr{P}(\mathcal{U})$ be a power set of $\mathcal{U}, k=\left\{k_{1}, k_{2}, k_{3}, \ldots, k_{n}\right\}(n \geq 1)$ be a set of attributes, and set $K_{i}$ be a set of corresponding subattributes of $k_{i}$, respectively, with $K_{i} \cap K_{j}=\varphi$ for $n \geq 1$ for each $i, j \in\{1,2,3, \ldots, n\}$, and $i \neq j$. Assume $K_{1} \times K_{2} \times$ $K_{3} \times \cdots \times K_{n}=\mathscr{A}=\left\{d_{1 h} \times d_{2 k} \times \cdots \times d_{n l}\right\}$ be a collection of multiattributes, where $1 \leq h \leq \alpha, 1 \leq k \leq \beta$, and $1 \leq l \leq \gamma$ and $\alpha$, $\beta$, and $\gamma \in \mathbb{N}$. Then, the pair $\left(\mathscr{F}, K_{1} \times K_{2} \times K_{3} \times \cdots \times K_{n}=\right.$ $\mathscr{A})$ is said to be HSS over $\mathcal{U}$ and its mapping is defined as

$$
\mathscr{F}: K_{1} \times K_{2} \times K_{3} \times \cdots \times K_{n}=\stackrel{\mathscr{A}}{\longrightarrow} \mathscr{P}(\mathcal{U}) .
$$

It is also defined as

$$
(\mathscr{F}, \ddot{\mathscr{A}})=\left\{\check{d}, \mathscr{F}_{\mathscr{A}}(\check{d}): \check{d} \in \check{\mathscr{A}}, \mathscr{F}_{\mathscr{A}}(\check{d}) \in \mathscr{P}(\mathscr{U})\right\} .
$$

Definition 4 (see [52]). Let $\mathcal{U}$ be a universe of discourse, $\mathscr{P}(\mathcal{U})$ be a power set of $\mathcal{U}$, and $k=\left\{k_{1}, k_{2}, k_{3}, \ldots, k_{n}\right\}$ $(n \geq 1)$ be a set of attributes, and set $K_{i}$ be a set of corresponding subattributes of $k_{i}$, respectively, with $K_{i} \cap K_{j}=\varphi$ for $n \geq 1$ for each $i, j \in\{1,2,3, \ldots, n\}$, and $i \neq j$. Assume $K_{1} \times$ $K_{2} \times K_{3} \times \cdots \times K_{n}=\mathscr{A}=\left\{d_{1 h} \times d_{2 k} \times \cdots \times d_{n l}\right\}$ be a collection of subattributes, where $1 \leq h \leq \alpha, 1 \leq k \leq \beta$, and $1 \leq l \leq \gamma$, and $\alpha, \beta$, and $\gamma \in \mathbb{N}_{\text {and }} \mathbb{F}^{\mathcal{U}}$ be a collection of all fuzzy subsets over $\mathcal{U}$. Then, the pair $\left(\mathscr{F}, K_{1} \times K_{2} \times K_{3} \times \cdots \times K_{n}=\mathscr{A}\right)$ is said to be FHSS over $\mathcal{U}$ and its mapping is defined as

$$
\mathscr{F}: K_{1} \times K_{2} \times K_{3} \times \cdots \times K_{n}=\cdots \mathscr{A} \longrightarrow \mathbb{F}^{\mathscr{U}} \text {. }
$$

It is also defined as

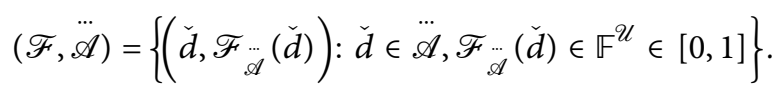

Example 1. Consider the universe of discourse $\mathscr{U}=\left\{\delta_{1}, \delta_{2}\right\}$ and $\mathscr{L}=\left\{\ell_{1}=\right.$ Teaching methdology, $\ell_{2}=$ Subjects, $\ell_{3}=$ Classes $\}$ be a collection of attributes with their corresponding subattribute values given as teaching methodology $=L_{1}=\left\{d_{11}=\right.$ project base, $\quad d_{12}=$ class discussion $\}$, subjects $=L_{2}=\left\{d_{21}=\right.$ Mathematics, $d_{22}=$ Computer Science Science, $d_{23}=$ Statistics $\}$ and classes $=$ $L_{3}=\left\{d_{31}=\right.$ Masters, $d_{32}=$ Doctorol $\}$. Let $\mathscr{A}=L_{1} \times L_{2} \times L_{3}$ be a set of attributes:

$$
\begin{aligned}
\ddot{\mathscr{A}} & =L_{1} \times L_{2} \times L_{3}=\left\{d_{11}, d_{12}\right\} \times\left\{d_{21}, d_{22}, d_{23}\right\} \times\left\{d_{31}, d_{32}\right\} \\
& =\left\{\begin{array}{l}
\left(d_{11}, d_{21}, d_{31}\right),\left(d_{11}, d_{21}, d_{32}\right),\left(d_{11}, d_{22}, d_{31}\right),\left(d_{11}, d_{22}, d_{32}\right),\left(d_{11}, d_{23}, d_{31}\right),\left(d_{11}, d_{23}, d_{32}\right), \\
\left(d_{12}, d_{21}, d_{31}\right),\left(d_{12}, d_{21}, d_{32}\right),\left(d_{12}, d_{22}, d_{31}\right),\left(d_{12}, d_{22}, d_{32}\right),\left(d_{12}, d_{23}, d_{31}\right),\left(d_{12}, d_{23}, d_{32}\right),
\end{array}\right\} \\
\ddot{\mathscr{A}} & =\left\{\check{d}_{1}, \check{d}_{2}, \check{d}_{3}, \check{d}_{4}, \check{d}_{5}, \check{d}_{6}, \check{d}_{7}, \check{d}_{8}, \check{d}_{9}, \check{d}_{10}, \check{d}_{11}, \check{d}_{12}\right\} .
\end{aligned}
$$


Then, the FHSS over $\mathcal{U}$ is given as follows:

$$
(\mathscr{F}, \ldots)=\left\{\begin{aligned}
\left(\check{d}_{1},\left(\delta_{1}, 0.6\right),\left(\delta_{2}, 0.3\right)\right),\left(\check{d}_{2},\left(\delta_{1}, 0.7\right),\left(\delta_{2}, 0.5\right)\right),\left(\check{d}_{3},\left(\delta_{1}, 0.8\right),\left(\delta_{2}, 0.3\right)\right),\left(\check{d}_{4},\left(\delta_{1}, 0.2\right),\left(\delta_{2}, 0.8\right)\right), \\
\left(\check{d}_{5},\left(\delta_{1}, 0.4\right),\left(\delta_{2}, 0.3\right)\right),\left(\check{d}_{6},\left(\delta_{1}, 0.2\right),\left(\delta_{2}, 0.5\right)\right),\left(\check{d}_{7},\left(\delta_{1}, 0.6\right),\left(\delta_{2}, 0.9\right)\right),\left(\check{d}_{8},\left(\delta_{1}, 0.2\right),\left(\delta_{2}, 0.8\right)\right), \\
\left(\check{d}_{9},\left(\delta_{1}, 0.4\right),\left(\delta_{2}, 0.7\right)\right),\left(\check{d}_{10},\left(\delta_{1}, 0.1\right),\left(\delta_{2}, 0.7\right)\right),\left(\check{d}_{11},\left(\delta_{1}, 0.4\right),\left(\delta_{2}, 0.6\right)\right),\left(\check{d}_{12},\left(\delta_{1}, 0.2\right),\left(\delta_{2}, 0.7\right)\right)
\end{aligned}\right\} .
$$

Definition 5. (see [52]). Let $\mathscr{U}$ be a universe of discourse, $\mathscr{P}(\mathcal{U})$ be a power set of $\mathscr{U}$, and $k=\left\{k_{1}, k_{2}, k_{3}, \ldots, k_{n}\right\}(n \geq 1)$ be a set of attributes, and set $K_{i}$ be a set of corresponding subattributes of $k_{i}$, respectively, with $K_{i} \cap K_{j}=\varphi$ for $n \geq 1$ for each $i, j \in\{1,2,3, \ldots, n\}$, and $i \neq j$. Assume $K_{1} \times K_{2} \times K_{3} \times$ $\cdots \times K_{n}=\ddot{\mathscr{A}}=\left\{d_{1 h} \times d_{2 k} \times \cdots \times d_{n l}\right\}$ be a collection of subattributes, where $1 \leq h \leq \alpha, 1 \leq k \leq \beta$, and $1 \leq l \leq \gamma$, and $\alpha, \beta$, and $\gamma \in \mathbb{N}$ and IFS $^{\mathscr{U}}$ be a collection of all intuitionistic fuzzy subsets over $\mathscr{U}$. Then, the pair $\left(\mathscr{F}, K_{1} \times K_{2} \times K_{3} \times \cdots \times K_{n}=\right.$ $\ddot{\mathscr{A}})$ is said to be IFHSS over $\mathcal{U}$, and its mapping is defined as

$$
\mathscr{F}: K_{1} \times K_{2} \times K_{3} \times \cdots \times K_{n}=\ddot{\mathscr{A}} \longrightarrow \mathrm{IFS}^{\mathscr{U}} .
$$

It is also defined as $(\mathscr{F}, \ddot{\mathscr{A}})=\{(\check{d}, \mathscr{F} \ldots(\check{d})): \check{d} \in \ddot{\mathscr{A}}, \mathscr{F} \ldots$ $\left.(\check{d}) \in \operatorname{IFS}^{\mathscr{U}} \in[0,1]\right\}$, where $\mathscr{F}_{\mathscr{A}}(\check{d})=\left\{\delta, \mathscr{T}_{\mathscr{F}(\check{d})}(\delta), \mathscr{J}_{\mathscr{F}(\check{d})}\right.$ $(\delta): \delta \in \mathscr{U}\}$, in which $\mathscr{T}_{\mathscr{F}(\check{d})}(\delta)$ and $\mathscr{J}_{\mathscr{F}(\check{d})}(\delta)$ represent the membership and nonmembership values of the attributes such as $\mathscr{T}_{\mathscr{F}(\check{d})}(\delta), \mathscr{J}_{\mathscr{F}(\check{d})}(\delta) \in \in[0,1]$, and $0 \leq \mathscr{T}_{\mathscr{F}(\check{d})}(\delta)+$ $\mathscr{J}_{\mathscr{F}(\check{d})}(\delta) \leq 1$.

Simply an intuitionistic fuzzy hypersoft number (IFHSN) can be expressed as $\mathscr{F}=\left\{\left(\mathscr{T}_{\mathscr{F}(\check{d})}(\delta), \mathscr{J}_{\mathscr{F}(\check{d})}(\delta)\right)\right\}$, where $0 \leq \mathscr{T}_{\mathscr{F}(\breve{d})}(\delta)+\mathscr{J}_{\mathscr{F}(\breve{d})}(\delta) \leq 1$.

Example 2. Consider the universe of discourse $\mathscr{U}=\left\{\delta_{1}, \delta_{2}\right\}$ and $\mathscr{L}=\left\{\ell_{1}=\right.$ Teaching methdology, $\ell_{2}=$ Subjects, $\ell_{3}=$ Classes $\}$ be a collection of attributes with their corresponding attribute values given as teaching methodology $=$ $L_{1}=\left\{d_{11}=\right.$ project base, $d_{12}=$ class discussion $\}$, subjects $=$ $L_{2}=\left\{d_{21}=\right.$ Mathematics, $d_{22}=$ Computer Science, $\quad d_{23}=$ Statistics $\}$, and classes $=L_{3}=\left\{d_{31}=\right.$ Masters, $d_{32}=$ Doctorol $\}$. Let $\mathscr{A}=L_{1} \times L_{2} \times L_{3}$ be a set of attributes:

$$
\begin{aligned}
\mathscr{A} & =L_{1} \times L_{2} \times L_{3}=\left\{d_{11}, d_{12}\right\} \times\left\{d_{21}, d_{22}, d_{23}\right\} \times\left\{d_{31}, d_{32}\right\} \\
& =\left\{\begin{array}{l}
\left(d_{11}, d_{21}, d_{31}\right),\left(d_{11}, d_{21}, d_{32}\right),\left(d_{11}, d_{22}, d_{31}\right),\left(d_{11}, d_{22}, d_{32}\right),\left(d_{11}, d_{23}, d_{31}\right),\left(d_{11}, d_{23}, d_{32}\right), \\
\left(d_{12}, d_{21}, d_{31}\right),\left(d_{12}, d_{21}, d_{32}\right),\left(d_{12}, d_{22}, d_{31}\right),\left(d_{12}, d_{22}, d_{32}\right),\left(d_{12}, d_{23}, d_{31}\right),\left(d_{12}, d_{23}, d_{32}\right),
\end{array}\right\} \\
\dddot{\mathscr{A}} & =\left\{\check{d}_{1}, \check{d}_{2}, \check{d}_{3}, \check{d}_{4}, \check{d}_{5}, \check{d}_{6}, \check{d}_{7}, \check{d}_{8}, \check{d}_{9}, \check{d}_{10}, \check{d}_{11}, \check{d}_{12}\right\} .
\end{aligned}
$$

Then, the IFHSS over $\mathscr{U}$ is given as follows:

$$
(\mathscr{F}, \ddot{\mathscr{A}})=\left\{\begin{aligned}
\left(\check{d}_{1},\left(\delta_{1},(0.6,0.3)\right),\left(\delta_{2},(0.3,0.5)\right)\right),\left(\check{d}_{2},\left(\delta_{1},(0.2,0.7)\right),\left(\delta_{2},(0.1,0.5)\right)\right),\left(\check{d}_{3},\left(\delta_{1},(0.2,0.8)\right),\left(\delta_{2},(0.3,0.4)\right)\right), \\
\left(\check{d}_{4},\left(\delta_{1},(0.2,0.5)\right),\left(\delta_{2},(0.1,0.6)\right)\right),\left(\check{d}_{5},\left(\delta_{1},(0.4,0.3)\right),\left(\delta_{2},(0.3,0.5)\right)\right),\left(\check{d}_{6},\left(\delta_{1},(0.2,0.4)\right),\left(\delta_{2},(0.1,0.5)\right)\right), \\
\left(\check{d}_{7},\left(\delta_{1},(0.2,0.6)\right),\left(\delta_{2},(0.4,0.2)\right)\right),\left(\check{d}_{8},\left(\delta_{1},(0.2,0.5)\right),\left(\delta_{2},(0.3,0.1)\right)\right),\left(\check{d}_{9},\left(\delta_{1},(0.4,0.2)\right),\left(\delta_{2},(0.3,0.5)\right)\right), \\
\left(\check{d}_{10},\left(\delta_{1},(0.1,0.4)\right),\left(\delta_{2},(0.7,0.2)\right)\right),\left(\check{d}_{11},\left(\delta_{1},(0.4,0.5)\right),\left(\delta_{2},(0.2,0.5)\right)\right),\left(\check{d}_{5},\left(\delta_{1},(0.1,0.2)\right),\left(\delta_{2},(0.2,0.7)\right)\right)
\end{aligned}\right\} .
$$

The abovementioned IFHSS cannot deal with the situation when the sum of MD and NMD of subattributes exceeds one, so to handle such situations in this paper, we introduce the general notion of PFHSS with its characteristics by modifying the condition $\mathrm{MD}+\mathrm{NMD} \leq 1$ to $\mathrm{MD}^{2}+\mathrm{NMD}^{2} \leq 1$

Definition 6. Let $\mathcal{U}$ be a universe of discourse, $\mathscr{P}(\mathcal{U})$ be a power set of $\mathscr{U}, k=\left\{k_{1}, k_{2}, k_{3}, \ldots, k_{n}\right\}(n \geq 1)$ be a set of attributes, and set $K_{i}$ be a set of corresponding subattributes of $k_{i}$, respectively, with $K_{i} \cap K_{j}=\varphi$ for $n \geq 1$ for each $i, j \in\{1,2,3, \ldots, n\}$ and $i \neq j$. Assume $K_{1} \times K_{2} \times K_{3} \times \cdots \times$ $K_{n}=\ddot{\mathscr{A}}=\left\{d_{1 h} \times d_{2 k} \times \cdots \times d_{n l}\right\}$ be a collection of subattributes, where $1 \leq h \leq \alpha, 1 \leq k \leq \beta$, and $1 \leq l \leq \gamma$, and $\alpha$, $\beta$, and $\gamma \in \mathbb{N}$, and PFS $^{\mathscr{U}}$ be a collection of all Pythagorean fuzzy subsets over $\mathcal{U}$. Then, the pair $\left(\mathscr{F}, K_{1} \times K_{2} \times K_{3} \times \cdots \times K_{n}=\mathscr{A}\right)$ is said to be PFHSS over $\mathcal{U}$ and its mapping is defined as

$$
\mathscr{F}: K_{1} \times K_{2} \times K_{3} \times \cdots \times K_{n}=\ddot{\mathscr{A}} \longrightarrow \mathrm{PFS}^{\mathscr{U}} .
$$


It is also defined as $(\mathscr{F}, \ddot{A})=$ $\left\{\left(\check{d}, \mathscr{F}_{\mathscr{A}}(\check{d})\right): \check{d} \in \stackrel{\mathscr{A}}{\mathscr{A}} \mathscr{F}_{\mathscr{A}}(\check{d}) \in \operatorname{PFS}^{\mathscr{U}} \in[0,1]\right\}, \quad$ where $\mathscr{F}_{\mathscr{A}}(\check{d})=\left\{\delta, \mathscr{T}_{\mathscr{F}(\check{d})}(\delta), \mathscr{J}_{\mathscr{F}(\check{d})}(\delta): \delta \in \mathcal{U}\right\}, \quad$ in which $\mathscr{T}_{\mathscr{F}(\check{d})}(\delta)$ and $\mathscr{J}_{\mathscr{F}(\check{d})}(\delta)$ represent the membership and nonmembership values of the attributes such as $\mathscr{T}_{\mathscr{F}(\check{d})}(\delta)$, $\mathscr{J}_{\mathscr{F}(\check{d})}(\delta) \in \in[0,1]$, and $0 \leq\left(\mathscr{T}_{\mathscr{F}(\check{d})}(\delta)\right)^{2}+\left(\mathscr{F}_{\mathscr{F}(\check{d})}(\delta)\right)^{2} \leq 1$.

Simply, a Pythagorean fuzzy hypersoft number (PFHSN) can be expressed as $\mathscr{F}=\left\{\left(\mathscr{T}_{\mathscr{F}(\check{d})}(\delta), \mathscr{J}_{\mathscr{F}(\check{d})}(\delta)\right)\right\}$, where $0 \leq\left(\mathscr{T}_{\mathscr{F}(\check{d})}(\delta)\right)^{2}+\left(\mathscr{J}_{\mathscr{F}(\check{d})}(\delta)\right)^{2} \leq 1$.
Example 3. Consider the universe of discourse $\mathscr{U}=\left\{\delta_{1}, \delta_{2}\right\}$ and $\mathscr{L}=\left\{\ell_{1}=\right.$ Teaching methdology, $\ell_{2}=$ Subjects, $\ell_{3}=$ Classes $\}$ be a collection of attributes with their corresponding attribute values given as teaching methodology $=$ $L_{1}=\left\{d_{11}=\right.$ project base, $d_{12}=$ class discussion $\}$, subjects $=$ $L_{2}=\left\{d_{21}=\right.$ Mathematics, $d_{22}=$ Computer Science, $\quad d_{23}=$ Statistics $\}$, and classes $=L_{3}=\left\{d_{31}=\right.$ Masters, $\quad d_{32}=$ Doctorol $\}$. Let $\mathscr{A}=L_{1} \times L_{2} \times L_{3}$ be a set of attributes:

$$
\begin{aligned}
\mathscr{A} & =L_{1} \times L_{2} \times L_{3}=\left\{d_{11}, d_{12}\right\} \times\left\{d_{21}, d_{22}, d_{23}\right\} \times\left\{d_{31}, d_{32}\right\} \\
& =\left\{\begin{array}{l}
\left(d_{11}, d_{21}, d_{31}\right),\left(d_{11}, d_{21}, d_{32}\right),\left(d_{11}, d_{22}, d_{31}\right),\left(d_{11}, d_{22}, d_{32}\right),\left(d_{11}, d_{23}, d_{31}\right),\left(d_{11}, d_{23}, d_{32}\right), \\
\left(d_{12}, d_{21}, d_{31}\right),\left(d_{12}, d_{21}, d_{32}\right),\left(d_{12}, d_{22}, d_{31}\right),\left(d_{12}, d_{22}, d_{32}\right),\left(d_{12}, d_{23}, d_{31}\right),\left(d_{12}, d_{23}, d_{32}\right),
\end{array}\right\} \\
\cdots & =\left\{\check{d}_{1}, \check{d}_{2}, \check{d}_{3}, \check{d}_{4}, \check{d}_{5}, \check{d}_{6}, \check{d}_{7}, \check{d}_{8}, \check{d}_{9}, \check{d}_{10}, \check{d}_{11}, \check{d}_{12}\right\} .
\end{aligned}
$$

Then, the PFHSS over $\mathcal{U}$ is given as follows:

$$
(\mathscr{F}, \mathscr{A})=\left\{\begin{aligned}
&\left(\check{d}_{1},\left(\delta_{1},(0.6,0.3)\right),\left(\delta_{2},(0.3,0.5)\right)\right),\left(\check{d}_{2},\left(\delta_{1},(0.2,0.7)\right),\left(\delta_{2},(0.1,0.5)\right)\right),\left(\check{d}_{3},\left(\delta_{1},(0.2,0.8)\right),\left(\delta_{2},(0.3,0.7)\right)\right), \\
&\left(\check{d}_{4},\left(\delta_{1},(0.6,0.5)\right),\left(\delta_{2},(0.5,0.6)\right)\right),\left(\check{d}_{5},\left(\delta_{1},(0.7,0.3)\right),\left(\delta_{2},(0.4,0.8)\right)\right),\left(\check{d}_{6},\left(\delta_{1},(0.5,0.4)\right),\left(\delta_{2},(0.6,0.5)\right)\right), \\
&\left(\check{d}_{7},\left(\delta_{1},(0.6,0.5)\right),\left(\delta_{2},(0.5,0.6)\right)\right),\left(\check{d}_{5},\left(\delta_{1},(0.7,0.3)\right),\left(\delta_{2},(0.4,0.8)\right)\right),\left(\check{d}_{6},\left(\delta_{1},(0.5,0.4)\right),\left(\delta_{2},(0.6,0.5)\right)\right), \\
&\left(\check{d}_{10},\left(\delta_{1},(0.7,0.4)\right),\left(\delta_{2},(0.7,0.2)\right)\right),\left(\check{d}_{11},\left(\delta_{1},(0.4,0.5)\right),\left(\delta_{2},(0.5,0.3)\right)\right),\left(\check{d}_{12},\left(\delta_{1},(0.5,0.7)\right),\left(\delta_{2},(0.4,0.7)\right)\right)
\end{aligned}\right\} .
$$

Remark 1.

(1) If both $\left(\mathscr{T}_{\mathscr{F}(\check{d})}(\delta)\right)^{2}+\left(\mathscr{F}_{\mathscr{F}(\check{d})}(\delta)\right)^{2} \leq 1 \quad$ and $\mathscr{T}_{\mathscr{F}(\check{d})}(\delta)+\mathscr{J}_{\mathscr{F}(\check{d})}(\delta) \leq 1$ hold, then PFHSS was reduced to IFHSS [56].

(2) If $\left(\mathscr{T}_{\mathscr{F}(\check{d})}(\delta)\right)^{2}+\left(\mathscr{J}_{\mathscr{F}(\check{d})}(\delta)\right)^{2} \leq 1$ and each parameter of the set of attributes consists of no subattribute, then PFHSS was reduced to PFSS [31].

(3) If both $\left(\mathscr{T}_{\mathscr{F}(\check{d})}(\delta)\right)^{2}+\left(\mathscr{F}_{\mathscr{F}(\check{d})}(\delta)\right)^{2} \leq 1 \quad$ and $\mathscr{T}_{\mathscr{F}(\check{d})}(\delta)+\mathscr{J}_{\mathscr{F}(\check{d})}(\delta) \leq 1$ hold and a set of attributes contains only one parameter with no subattributes, then PFHSS was reduced to IFSS [22].

For simplicity, we will express $\mathscr{F}_{\delta_{i}}\left(\check{d}_{j}\right)=\left\{\left(\mathscr{T} \mathscr{F}\left(\check{d}_{j}\right)\right.\right.$ $\left.\left.\left(\delta_{i}\right), \mathscr{J}_{\mathscr{F}\left(\check{d}_{j}\right)}\left(\delta_{i}\right)\right) \mid \delta_{i} \in \mathcal{U}\right\}$ as $\widetilde{\mho}_{\check{d}_{i j}}=\mathscr{T}_{\mathscr{F}\left(\check{d}_{i j}\right)}$, and $\mathscr{F}_{\mathscr{F}\left(\check{d}_{i j}\right)}$ is called PFHSN. In the process of applying PFHSNs in actual problems, it is essential to rank them. For this, the scoring function of $\mathfrak{J}_{\check{d}_{i j}}$ is defined as follows:

$$
\mathbb{S}\left(\widetilde{J}_{\check{d}_{i j}}\right)=\mathscr{T}_{\mathscr{F}\left(\check{d}_{i j}\right)}^{2}-\mathscr{J}_{\mathscr{F}\left(\check{d}_{i j}\right)}^{2}, \quad \mathbb{S}\left(\mathfrak{J}_{\check{d}_{i j}}\right) \in[-1,1] .
$$

However, in some cases, the scoring function cannot compare the two PFHSNs. such as $\widetilde{J}_{\check{d}_{11}}=\langle 0.4,0.7\rangle$ and $\mathfrak{\Im}_{\check{d}_{12}}=\langle 0.5568,0.8\rangle$, and it is impossible to know which is greater because $\mathbb{S}\left(\mathfrak{J}_{\check{d}_{11}}\right)=0.33=\mathbb{S}\left(\mathfrak{\Im}_{\check{d}_{12}}\right)$. For this, an accuracy function is defined as follows:

$$
H\left(\mathfrak{\Im}_{\check{d}_{i j}}\right)=\mathscr{T}_{\mathscr{F}\left(\check{d}_{i j}\right)}^{2}+\mathscr{J}_{\mathscr{F}\left(\check{d}_{i j}\right)}^{2}, \quad H\left(\mathfrak{J}_{\check{d}_{i j}}\right) \in[0,1] .
$$

Thus, to compare two PFHSNs $\mathfrak{\Im}_{\breve{d}_{i j}}$ and $\mathfrak{I}_{\breve{d}_{i j}}$, the ranking and comparison laws are defined as follows: 
(1) If $\mathbb{S}\left(\mathfrak{J}_{\breve{d}_{i j}}\right)>\mathbb{S}\left(\mathfrak{I}_{\check{d}_{i j}}\right)$, then $\mathfrak{J}_{\check{d}_{i j}}>\mathfrak{T}_{\check{d}_{i j}}$.

(2) If $\mathbb{S}\left(\mathfrak{J}_{\breve{d}_{i j}}\right)=\mathbb{S}\left(\mathfrak{I}_{\check{d}_{i j}}\right)$, then the following holds:

(i) If $H\left(\mathfrak{\Im}_{\check{d}_{i j}}\right)>H\left(\mathfrak{I}_{\check{d}_{i j}}\right)$, then $\mathfrak{J}_{\check{d}_{i j}}>\mathfrak{I}_{\check{d}_{i j}}$.

(ii) If $H\left(\mathfrak{J}_{d_{i j}}\right)=H\left(\mathfrak{I}_{\dot{d}_{i j}}\right)$, then $\mathfrak{J}_{d_{i j}}=\mathfrak{I}_{d_{i j}}$.

\section{Correlation Coefficient for Pythagorean Fuzzy Hypersoft Set}

In this section, the concept of correlation coefficient and weighted correlation coefficient and some aggregation operators on PFHSS have been proposed with some basic properties.

Definition 7. Let $(\mathscr{F}, \ddot{\mathscr{A}})=\left\{\left(\delta_{i}, \mathscr{T}_{\mathscr{F}\left(\check{d}_{k}\right)}\left(\delta_{i}\right), \mathscr{J}_{\mathscr{F}\left(\check{d}_{k}\right)}\left(\delta_{i}\right)\right) \mid\right.$ $\left.\delta_{i} \in \mathscr{U}\right\}$ and $(\mathscr{G}, \mathfrak{B})=\left\{\left(\delta_{i}, \mathscr{T}_{\mathscr{G}\left(\check{d}_{k}\right)}\left(\delta_{i}\right), \mathscr{J}_{\mathscr{G}\left(\check{(}_{k}\right)}\left(\delta_{i}\right)\right) \mid \delta_{i} \in \mathcal{U}\right\}$ be two PFHSSs defined over a universe of discourse $\mathcal{U}$. Then, their informational energies of $(\mathscr{F}, \ddot{\mathscr{A}})$ and $(\mathscr{G}, \ddot{B})$ can be described as follows:

$$
\begin{aligned}
\zeta_{\mathrm{PFHSS}}(\mathscr{F}, \ddot{\mathscr{A}}) & =\sum_{k=1}^{m} \sum_{i=1}^{n}\left(\left(\mathscr{T}_{\mathscr{F}\left(\check{d}_{k}\right)}\left(\delta_{i}\right)\right)^{4}+\left(\mathscr{F}_{\mathscr{F}\left(\check{d}_{k}\right)}\left(\delta_{i}\right)\right)^{4}\right), \\
\zeta_{\mathrm{PFHSS}}(\mathscr{G}, \mathfrak{B}) & =\sum_{k=1}^{m} \sum_{i=1}^{n}\left(\left(\mathscr{T}_{\mathscr{G}\left(\check{d}_{k}\right)}\left(\delta_{i}\right)\right)^{4}+\left(\mathscr{J}_{\mathscr{G}\left(\check{d}_{k}\right)}\left(\delta_{i}\right)\right)^{4}\right) .
\end{aligned}
$$

Definition 8. Let... $(\mathscr{F}, \ddot{\mathscr{A}})=\left\{\left(\delta_{i}, \mathscr{T}_{\mathscr{F}\left(\check{d}_{l}\right)}\left(\delta_{i}\right), \mathscr{J}_{\mathscr{F}\left(\check{d}_{k}\right)}\left(\delta_{i}\right)\right)\right\}$ $\left.\delta_{i} \in \mathscr{U}\right\}$ and $(\mathscr{G}, \mathfrak{B})=\left\{\left(\delta_{i}, \mathscr{T}_{\mathscr{G}\left(\check{d}_{k}\right)}\left(\delta_{i}\right), \mathscr{J}_{\mathscr{G}\left(\check{d}_{k}\right)}\left(\delta_{i}\right)\right) \mid \delta_{i} \in \mathscr{U}\right\}$ be two PFHSSs defined over a universe of discourse $\mathcal{U}_{. . \text {Then, }}$ their correlation measure between $(\mathscr{F}, \mathscr{A})$ and $(\mathscr{G}, \mathfrak{B})$ can be described as follows:

$$
\begin{aligned}
\mathscr{C}_{\mathrm{PFHSS}}((\mathscr{F}, \ddot{\mathscr{A}}),(\mathscr{G}, \dddot{\mathfrak{B}})) \\
=\sum_{k=1}^{m} \sum_{i=1}^{n}\left(\left(\mathscr{T}_{\mathscr{F}\left(d_{k}\right)}\left(\delta_{i}\right)\right)^{2} *\left(\mathscr{T}_{\mathscr{G}\left(\check{d}_{k}\right)}\left(\delta_{i}\right)\right)^{2}\right. \\
\left.\quad+\left(\mathscr{J}_{\mathscr{F}\left(\check{d}_{k}\right)}\left(\delta_{i}\right)\right)^{2} *\left(\mathscr{J}_{\mathscr{G}\left(\check{d}_{k}\right)}\left(\delta_{i}\right)\right)^{2}\right) .
\end{aligned}
$$

Theorem 1. Let $(\mathscr{F}, \ddot{\mathscr{A}})=\left\{\left(\delta_{i}, \mathscr{T}_{\mathscr{F}\left(\check{d}_{k}\right)}\left(\delta_{i}\right), \mathscr{J}_{\mathscr{F}\left(\check{d}_{k}\right)}\left(\delta_{i}\right)\right) \mid\right.$ $\left.\delta_{i} \in \mathscr{U}\right\}$ and $(\mathscr{G}, \dddot{B})=\left\{\left(\delta_{i}, \mathscr{T}_{\mathscr{G}\left(\check{d}_{k}\right)}\left(\delta_{i}\right), \mathscr{J}_{\mathscr{G}\left(\check{d}_{k}\right)}\left(\delta_{i}\right)\right) \mid \delta_{i} \in \mathscr{U}\right\}$ be two $\mathscr{C}_{\text {PFHSSSS }}$ and $\mathscr{C}_{\text {IFHSS }}((\mathscr{F}, \mathscr{A}),(\mathscr{G}, \mathfrak{B}))$ be a correlation between them, then the following properties hold:

(1) $\mathscr{C}_{\text {PFHSS }}((\mathscr{F}, \ddot{\mathscr{A}}),(\mathscr{F}, \ddot{\mathscr{A}}))=\varsigma_{\text {PFHSS }}(\mathscr{F}, \ddot{\mathscr{A}})$.

(2) $\mathscr{C}_{\text {PFHSS }}((\mathscr{G}, \mathfrak{B}),(\mathscr{G}, \mathfrak{B}))=\varsigma_{\text {PFHSS }}(\mathscr{G}, \mathfrak{B})$.

Proof. The proof is trivial.

Definition 9. Let $(\mathscr{F}, \dddot{\mathscr{A}})=\left\{\left(\delta_{i}, \mathscr{T}_{\mathscr{F}\left(\check{d}_{k}\right)}\left(\delta_{i}\right), \mathscr{J}_{\mathscr{F}\left(\check{d}_{k}\right)}\left(\delta_{i}\right)\right) \mid\right.$ $\left.\delta_{i} \in \mathscr{U}\right\}$ and $(\mathscr{G}, \ddot{B})=\left\{\left(\delta_{i}, \mathscr{T}_{\mathscr{G}\left(\check{d}_{k}\right)}\left(\delta_{i}\right), \mathscr{J}_{\mathscr{G}\left(\check{d}_{k}\right)}\left(\delta_{i}\right)\right) \mid \delta_{i} \in \mathcal{U}\right\}$ be two PFHSSs, then correlation coefficient between them is given as $\delta_{\text {PFHSS }}((\mathscr{F}, \ddot{A}),(\mathscr{G}, \ddot{\mathfrak{B}}))$ and is expressed as follows:

$$
\begin{aligned}
& \delta_{\mathrm{PFHSS}}((\mathscr{F}, \cdots),(\mathscr{G}, \ddot{\mathfrak{B}}))=\frac{\mathscr{C}_{\mathrm{PFHSS}}((\mathscr{F}, \mathscr{\mathscr { A }}),(\mathscr{G}, \ddot{\mathfrak{B}}))}{\sqrt{\varsigma_{\mathrm{PFHSS}}(\mathscr{F}, \ddot{A})} * \sqrt{\varsigma_{\mathrm{PFHSS}}(\mathscr{G}, \mathfrak{B})}} \\
& \delta_{\mathrm{PFHSS}}((\mathscr{F}, \ddot{\mathscr{A}}),(\mathscr{G}, \ddot{\mathfrak{B}}))=\frac{\sum_{k=1}^{m} \sum_{i=1}^{n}\left(\left(\mathscr{T}_{\mathscr{F}\left(d_{k}\right)}\left(\delta_{i}\right)\right)^{2} *\left(\mathscr{T}_{\mathscr{G}\left(\check{d}_{k}\right)}\left(\delta_{i}\right)\right)^{2}+\left(\mathscr{J}_{\mathscr{F}\left(\check{d}_{k}\right)}\left(\delta_{i}\right)\right)^{2} *\left(\mathscr{J}_{\mathscr{G}\left(\check{d}_{k}\right)}\left(\delta_{i}\right)\right)^{2}\right)}{\sqrt{\sum_{k=1}^{m} \sum_{i=1}^{n}\left(\left(\mathscr{T}_{\mathscr{F}\left(d_{k}\right)}\left(\delta_{i}\right)\right)^{4}+\left(\mathscr{J}_{\mathscr{F}\left(\check{d}_{k}\right)}\left(\delta_{i}\right)\right)^{4}\right)} \sqrt{\sum_{k=1}^{m} \sum_{i=1}^{n}\left(\left(\mathscr{T}_{\mathscr{G}\left(\check{d}_{k}\right)}\left(\delta_{i}\right)\right)^{4}+\left(\mathscr{J}_{\mathscr{G}\left(\check{d}_{k}\right)}\left(\delta_{i}\right)\right)^{4}\right)}} .
\end{aligned}
$$

Theorem 2. Let $(\mathscr{F}, \ddot{\mathscr{A}})=\left\{\left(\delta_{i}, \mathscr{T}_{\mathscr{F}\left(\check{d}_{k}\right)}\left(\delta_{i}\right), \mathscr{J}_{\mathscr{F}\left(\check{d}_{k}\right)}\left(\delta_{i}\right)\right) \mid\right.$ $\left.\delta_{i} \in \mathscr{U}\right\}$ and $(\mathscr{G}, \dddot{\mathfrak{B}})=\left\{\left(\delta_{i}, \mathscr{T}_{\mathscr{G}\left(\check{d}_{k}\right)}\left(\delta_{i}\right), \mathscr{J}_{\mathscr{G}\left(\check{d}_{k}\right)}\left(\delta_{i}\right)\right) \mid \delta_{i} \in \mathcal{U}\right\}$ be two PFHSSs, then CC between them satisfies the following properties:

(1) $0 \leq \delta_{\text {PFHSS }}((\mathscr{F}, \mathscr{A}),(\mathscr{G}, \mathfrak{B})) \leq 1$.

(2) $\delta_{\text {PFHSS }}((\mathscr{F}, \mathscr{A}),(\mathscr{G}, \mathfrak{B}))=\delta_{\text {PFHSS }}((\mathscr{G}, \mathfrak{B}),(\mathscr{F}, \ddot{\mathscr{A}}))$.
(3) If $(\mathscr{F}, \ddot{\mathscr{A}})=(\mathscr{G}, \ddot{\mathfrak{B}})$, that $i s, \quad \forall i, \quad k, \quad \mathscr{T}_{\mathscr{F}\left(\check{d}_{k}\right)}$ $\left(\delta_{i}\right)=\mathscr{T}_{\mathscr{G}\left(\check{d}_{k}\right)}\left(\delta_{i}\right)$, and $\mathscr{J}_{\mathscr{F}\left(\check{d}_{k}\right)}\left(\delta_{i}\right)=\mathscr{J}_{\mathscr{G}\left(\check{d}_{k}\right)}\left(\delta_{i}\right)$, then $\delta_{\text {PFHSS }}((\mathscr{F}, \ddot{\mathscr{A}}),(\mathscr{G}, \ddot{B}))=1$.

Proof. 1. $\delta_{\text {PFHSS }}((\mathscr{F}, \dddot{\mathscr{A}}),(\mathscr{G}, \mathfrak{B})) \geq 0$ is trivial, and here, we only need to prove that $\delta_{\text {PFHSS }}((\mathscr{F}, \ddot{\mathscr{A}}),(\mathscr{G}, \ddot{\mathfrak{B}})) \leq 1$. 
From Equation (18), we have

$$
\begin{aligned}
& \mathscr{C}_{\text {PFHSS }}((\mathscr{F}, \ddot{A}),(\mathscr{G}, \ddot{\mathfrak{B}}))=\sum_{k=1}^{m} \sum_{i=1}^{n}\left(\left(\mathscr{T}_{\mathscr{F}\left(\check{d}_{k}\right)}\left(\delta_{i}\right)\right)^{2} *\left(\mathscr{T}_{\mathscr{G}\left(\check{d}_{k}\right)}\left(\delta_{i}\right)\right)^{2}+\left(\mathscr{J}_{\mathscr{F}\left(\check{d}_{k}\right)}\left(\delta_{i}\right)\right)^{2} *\left(\mathscr{J}_{\mathscr{G}\left(\check{d}_{k}\right)}\left(\delta_{i}\right)\right)^{2}\right) \\
& =\sum_{k=1}^{m}\left(\left(\mathscr{T}_{\mathscr{F}\left(\check{d}_{k}\right)}\left(\delta_{1}\right)\right)^{2} *\left(\mathscr{T}_{\mathscr{G}\left(\check{d}_{k}\right)}\left(\delta_{1}\right)\right)^{2}+\left(\mathscr{J}_{\mathscr{F}\left(\check{d}_{k}\right)}\left(\delta_{1}\right)\right)^{2} *\left(\mathscr{J}_{\mathscr{G}\left(\check{d}_{k}\right)}\left(\delta_{1}\right)\right)^{2}\right) \\
& +\sum_{k=1}^{m}\left(\left(\mathscr{T}_{\mathscr{F}\left(\check{d}_{k}\right)}\left(\delta_{2}\right)\right)^{2} *\left(\mathscr{T}_{\mathscr{G}\left(\check{d}_{k}\right)}\left(\delta_{2}\right)\right)^{2}+\left(\mathscr{J}_{\mathscr{F}\left(\check{d}_{k}\right)}\left(\delta_{2}\right)\right)^{2} *\left(\mathscr{J}_{\mathscr{G}\left(\check{d}_{k}\right)}\left(\delta_{2}\right)\right)^{2}\right) \\
& + \\
& \text { : } \\
& + \\
& \cdot \sum_{k=1}^{m}\left(\left(\mathscr{T}_{\mathscr{F}\left(\check{d}_{k}\right)}\left(\delta_{n}\right)\right)^{2} *\left(\mathscr{T}_{\mathscr{G}\left(\check{d}_{k}\right)}\left(\delta_{n}\right)\right)^{2}+\left(\mathscr{J}_{\mathscr{F}\left(\check{d}_{k}\right)}\left(\delta_{n}\right)\right)^{2} *\left(\mathscr{J}_{\mathscr{G}\left(\check{d}_{k}\right)}\left(\delta_{n}\right)\right)^{2}\right) \\
& \mathscr{C}_{\text {PFHSS }}((\mathscr{F}, \mathscr{A}),(\mathscr{G}, \ldots))=\left\{\begin{array}{c}
\left(\mathscr{T}_{\mathscr{F}\left(\check{d}_{1}\right)}\left(\delta_{1}\right)\right)^{2} *\left(\mathscr{T}_{\mathscr{G}\left(\check{d}_{1}\right)}\left(\delta_{1}\right)\right)^{2}+\left(\mathscr{J}_{\mathscr{F}\left(\check{d}_{1}\right)}\left(\delta_{1}\right)\right)^{2} *\left(\mathscr{J}_{\mathscr{G}\left(\check{d}_{1}\right)}\left(\delta_{1}\right)\right)^{2}+ \\
\left(\mathscr{T}_{\mathscr{F}\left(\check{d}_{2}\right)}\left(\delta_{1}\right)\right)^{2} *\left(\mathscr{T}_{\mathscr{G}\left(\check{d}_{2}\right)}\left(\delta_{1}\right)\right)^{2}+\left(\mathscr{J}_{\mathscr{F}\left(\check{d}_{2}\right)}\left(\delta_{1}\right)\right)^{2} *\left(\mathscr{J}_{\mathscr{G}\left(\check{d}_{2}\right)}\left(\delta_{1}\right)\right)^{2}+ \\
\vdots \\
\left(\mathscr{T}_{\mathscr{F}\left(\check{d}_{m}\right)}\left(\delta_{1}\right)\right)^{2} *\left(\mathscr{T}_{\mathscr{G}\left(\check{d}_{m}\right)}\left(\delta_{1}\right)\right)^{2}+\left(\mathscr{J}_{\mathscr{F}\left(\check{d}_{m}\right)}\left(\delta_{1}\right)\right)^{2} *\left(\mathscr{J}_{\mathscr{G}\left(\check{d}_{m}\right)}\left(\delta_{1}\right)\right)^{2}
\end{array}\right\} \\
& +\left\{\begin{array}{c}
\left(\mathscr{T}_{\mathscr{F}\left(\check{d}_{1}\right)}\left(\delta_{2}\right)\right)^{2} *\left(\mathscr{T}_{\mathscr{G}\left(\check{d}_{1}\right)}\left(\delta_{2}\right)\right)^{2}+\left(\mathscr{J}_{\mathscr{F}\left(\check{d}_{1}\right)}\left(\delta_{2}\right)\right)^{2} *\left(\mathscr{J}_{\mathscr{G}\left(\check{d}_{1}\right)}\left(\delta_{2}\right)\right)^{2}+ \\
\left(\mathscr{T}_{\mathscr{F}\left(\check{d}_{2}\right)}\left(\delta_{2}\right)\right)^{2} *\left(\mathscr{T}_{\mathscr{G}\left(\check{d}_{2}\right)}\left(\delta_{2}\right)\right)^{2}+\left(\mathscr{J}_{\mathscr{F}\left(\check{d}_{2}\right)}\left(\delta_{2}\right)\right)^{2} *\left(\mathscr{J}_{\mathscr{G}\left(\check{d}_{2}\right)}\left(\delta_{2}\right)\right)^{2}+ \\
\vdots \\
\left(\mathscr{T}_{\mathscr{F}\left(\check{d}_{m}\right)}\left(\delta_{2}\right)\right)^{2} *\left(\mathscr{T}_{\mathscr{G}\left(\check{d}_{m}\right)}\left(\delta_{2}\right)\right)^{2}+\left(\mathscr{J}_{\mathscr{F}\left(\check{d}_{m}\right)}\left(\delta_{2}\right)\right)^{2} *\left(\mathscr{J}_{\mathscr{G}\left(\check{d}_{m}\right)}\left(\delta_{2}\right)\right)^{2}
\end{array}\right\} \\
& + \\
& \text { : } \\
& + \\
& \cdot\left\{\begin{array}{c}
\left(\mathscr{T}_{\mathscr{F}\left(\check{d}_{1}\right)}\left(\delta_{n}\right)\right)^{2} *\left(\mathscr{T}_{\mathscr{G}\left(\check{d}_{1}\right)}\left(\delta_{n}\right)\right)^{2}+\left(\mathscr{J}_{\mathscr{F}\left(\check{d}_{1}\right)}\left(\delta_{n}\right)\right)^{2} *\left(\mathscr{J}_{\mathscr{G}\left(\check{d}_{1}\right)}\left(\delta_{n}\right)\right)^{2}+ \\
\left(\mathscr{T}_{\mathscr{F}\left(\check{d}_{2}\right)}\left(\delta_{n}\right)\right)^{2} *\left(\mathscr{T}_{\mathscr{G}\left(\check{d}_{2}\right)}\left(\delta_{n}\right)\right)^{2}+\left(\mathscr{J}_{\mathscr{F}\left(\check{d}_{2}\right)}\left(\delta_{n}\right)\right)^{2} *\left(\mathscr{J}_{\mathscr{G}\left(\check{d}_{2}\right)}\left(\delta_{n}\right)\right)^{2}+ \\
\vdots \\
\left(\mathscr{T}_{\mathscr{F}\left(\check{d}_{m}\right)}\left(\delta_{n}\right)\right)^{2} *\left(\mathscr{T}_{\mathscr{G}\left(\check{d}_{m}\right)}\left(\delta_{n}\right)\right)^{2}+\left(\mathscr{J}_{\mathscr{F}\left(\check{d}_{m}\right)}\left(\delta_{n}\right)\right)^{2} *\left(\mathscr{J}_{\mathscr{G}\left(\check{d}_{m}\right)}\left(\delta_{n}\right)\right)^{2}
\end{array}\right\} \\
& =\sum_{k=1}^{m}\left(\left(\mathscr{T}_{\mathscr{F}\left(\check{d}_{k}\right)}\left(\delta_{1}\right)\right)^{2} *\left(\mathscr{T}_{\mathscr{G}\left(\check{d}_{k}\right)}\left(\delta_{1}\right)\right)^{2}+\left(\mathscr{T}_{\mathscr{F}\left(\check{d}_{k}\right)}\left(\delta_{2}\right)\right)^{2} *\left(\mathscr{T}_{\mathscr{G}\left(\check{d}_{k}\right)}\left(\delta_{2}\right)\right)^{2}\right. \\
& \left.+\ldots+\left(\mathscr{T}_{\mathscr{F}\left(\check{d}_{k}\right)}\left(\delta_{n}\right)\right)^{2} *\left(\mathscr{T}_{\mathscr{G}\left(\check{d}_{k}\right)}\left(\delta_{n}\right)\right)^{2}\right) \\
& +\sum_{k=1}^{m}\left(\left(\mathscr{J}_{\mathscr{F}\left(\check{d}_{k}\right)}\left(\delta_{1}\right)\right)^{2} *\left(\mathscr{J}_{\mathscr{G}\left(\check{d}_{k}\right)}\left(\delta_{1}\right)\right)^{2}+\left(\mathscr{J}_{\mathscr{F}\left(\check{d}_{k}\right)}\left(\delta_{2}\right)\right)^{2} *\left(\mathscr{J}_{\mathscr{G}\left(\check{d}_{k}\right)}\left(\delta_{2}\right)\right)^{2}\right. \\
& \left.+\cdots+\left(\mathscr{J}_{\mathscr{F}\left(\check{d}_{k}\right)}\left(\delta_{n}\right)\right)^{2} *\left(\mathscr{J}_{\mathscr{G}\left(\check{d}_{k}\right)}\left(\delta_{n}\right)\right)^{2}\right)
\end{aligned}
$$


Using Cauchy-Schwarz inequality, $\left(\alpha_{1} \beta_{1}+\alpha_{2} \beta_{2}+\cdots+\right.$ $\left.\alpha_{n} \beta_{n}\right)^{2} \leq\left(\alpha_{1}^{2}+\alpha_{2}^{2}+\cdots+\alpha_{n}^{2}\right) \cdot\left(\beta_{1}^{2}+\beta_{2}^{2}+\cdots+\beta_{n}^{2}\right)$, where $\left(\alpha_{1}+\alpha_{2}+\cdots+\alpha_{n}\right)$ and $\left(\beta_{1}+\beta_{2}+\cdots+\beta_{n}\right) \in \in \mathbb{R}^{n}$.

$$
\begin{aligned}
& \mathscr{C}_{\text {PFHSS }}((\mathscr{F}, \mathscr{A}),(\mathscr{G}, \mathfrak{B}))^{2} \\
& \leq \sum_{k=1}^{m}\left\{\left(\left(\mathscr{T}_{\mathscr{F}\left(\check{d}_{k}\right)}\left(\delta_{1}\right)\right)^{4}+\left(\mathscr{T}_{\mathscr{F}\left(\check{d}_{k}\right)}\left(\delta_{2}\right)\right)^{4}+\cdots+\left(\mathscr{T}_{\mathscr{F}\left(\check{d}_{k}\right)}\left(\delta_{n}\right)\right)^{4}\right)+\left(\left(\mathscr{F}_{\mathscr{F}\left(\check{d}_{k}\right)}\left(\delta_{1}\right)\right)^{4}+\left(\mathscr{F}_{\mathscr{F}\left(\check{d}_{k}\right)}\left(\delta_{2}\right)\right)^{4}+\cdots+\left(\mathscr{J}_{\mathscr{F}\left(\check{d}_{k}\right)}\left(\delta_{n}\right)\right)^{4}\right)\right\} \\
& \quad \times \sum_{k=1}^{m}\left\{\left(\left(\mathscr{T}_{\mathscr{G}\left(\check{d}_{k}\right)}\left(\delta_{1}\right)\right)^{4}+\left(\mathscr{T}_{\mathscr{G}\left(\check{d}_{k}\right)}\left(\delta_{2}\right)\right)^{4}+\cdots+\left(\mathscr{T}_{\mathscr{G}\left(\check{d}_{k}\right)}\left(\delta_{n}\right)\right)^{4}\right)+\left(\left(\mathscr{F}_{\mathscr{G}\left(\check{d}_{k}\right)}\left(\delta_{1}\right)\right)^{4}+\left(\mathscr{J}_{\mathscr{G}\left(\check{(}_{k}\right)}\left(\delta_{2}\right)\right)^{4}+\cdots+\left(\mathscr{J}_{\mathscr{G}\left(\check{d}_{k}\right)}\left(\delta_{n}\right)\right)^{4}\right)\right\}
\end{aligned}
$$$$
\mathscr{C}_{\mathrm{PFHSS}}((\mathscr{F}, \ddot{\mathscr{A}}),(\mathscr{G}, \ddot{\mathfrak{B}}))^{2}
$$$$
\leq \sum_{k=1}^{m} \sum_{i=1}^{n}\left(\left(\mathscr{T}_{\mathscr{F}\left(\check{d}_{k}\right)}\left(\delta_{i}\right)\right)^{4}+\left(\mathscr{J}_{\mathscr{F}\left(\check{d}_{k}\right)}\left(\delta_{i}\right)\right)^{4}\right) \times \sum_{k=1}^{m} \sum_{i=1}^{n}\left(\left(\mathscr{T}_{\mathscr{G}\left(\check{d}_{k}\right)}\left(\delta_{i}\right)\right)^{4}+\left(\mathscr{F}_{\mathscr{G}\left(\check{d}_{k}\right)}\left(\delta_{i}\right)\right)^{4}\right)
$$$$
\cdot \mathscr{C}_{\mathrm{PFHSS}}((\mathscr{F}, \ddot{\mathscr{A}}),(\mathscr{G}, \ddot{\mathfrak{B}}))^{2} \leq \varsigma_{\mathrm{PFHSS}}(\mathscr{F}, \ddot{\mathscr{A}}) \times \varsigma_{\mathrm{PFHSS}}(\mathscr{G}, \ddot{\mathfrak{B}}) .
$$

Therefore, $\quad \mathscr{C}_{\text {PFHSS }}((\mathscr{F}, \ddot{\mathscr{A}}),(\mathscr{G}, \mathfrak{B}))^{2} \leq \varsigma_{\text {PFHSS }}(\mathscr{F}, \ddot{\mathscr{A}}) \times \quad$ Proof. $\quad$ 2. The proof is obvious.

$\varsigma_{\text {PFHSS }}(\mathscr{G}, \dddot{\mathfrak{B}})$. Hence, by using Definition 9 , we have $\delta_{\text {PFHSS }}$ $((\mathscr{F}, \ddot{\mathscr{A}}),(\mathscr{G}, \ddot{\mathfrak{B}})) \leq 1$. So, $0 \leq \delta_{\text {PFHSS }}((\mathscr{F}, \ddot{\mathscr{A}}),(\mathscr{G}, \ddot{\mathfrak{B}})) \leq 1$.

Proof. 3. From Equation (19), we have

$\delta_{\mathrm{PFHSS}}((\mathscr{F}, \ddot{\mathscr{A}}),(\mathscr{G}, \ddot{\mathfrak{B}}))=\frac{\sum_{k=1}^{m} \sum_{i=1}^{n}\left(\left(\mathscr{T}_{\mathscr{F}\left(\check{d}_{k}\right)}\left(\delta_{i}\right)\right)^{2} *\left(\mathscr{T}_{\mathscr{G}\left(\check{d}_{k}\right)}\left(\delta_{i}\right)\right)^{2}+\left(\mathscr{J}_{\mathscr{F}\left(\check{d}_{k}\right)}\left(\delta_{i}\right)\right)^{2} *\left(\mathscr{J}_{\mathscr{G}\left(\check{d}_{k}\right)}\left(\delta_{i}\right)\right)^{2}\right)}{\sqrt{\sum_{k=1}^{m} \sum_{i=1}^{n}\left(\left(\mathscr{T}_{\mathscr{F}\left(\check{d}_{k}\right)}\left(\delta_{i}\right)\right)^{4}+\left(\mathscr{J}_{\mathscr{F}\left(\check{d}_{k}\right)}\left(\delta_{i}\right)\right)^{4}\right)} \sqrt{\sum_{k=1}^{m} \sum_{i=1}^{n}\left(\left(\mathscr{T}_{\mathscr{G}\left(\check{d}_{k}\right)}\left(\delta_{i}\right)\right)^{4}+\left(\mathscr{J}_{\mathscr{G}\left(\check{d}_{k}\right)}\left(\delta_{i}\right)\right)^{4}\right)}}$.

As we know that $\mathscr{T}_{\mathscr{F}\left(d_{k}\right)}\left(\delta_{i}\right)=\mathscr{T}_{\mathscr{G}\left(\check{d}_{k}\right)}\left(\delta_{i}\right)$ and $\mathscr{J}_{\mathscr{F}\left(\check{d}_{k}\right)}\left(\delta_{i}\right)=\mathscr{J}_{\mathscr{G}\left(\check{d}_{k}\right)}\left(\delta_{i}\right) \forall i, k$, we get

$\delta_{\mathrm{PFHSS}}((\mathscr{F}, \ddot{\mathscr{A}}),(\mathscr{G}, \ddot{\mathfrak{B}}))=\frac{\sum_{k=1}^{m} \sum_{i=1}^{n}\left(\left(\mathscr{T}_{\mathscr{F}\left(\check{d}_{k}\right)}\left(\delta_{i}\right)\right)^{4}+\left(\mathscr{F}_{\mathscr{F}\left(\check{d}_{k}\right)}\left(\delta_{i}\right)\right)^{4}\right)}{\sqrt{\sum_{k=1}^{m} \sum_{i=1}^{n}\left(\left(\mathscr{T}_{\mathscr{F}\left(\check{d}_{k}\right)}\left(\delta_{i}\right)\right)^{4}+\left(\mathscr{J}_{\mathscr{F}\left(\check{d}_{k}\right)}\left(\delta_{i}\right)\right)^{4}\right)} \sqrt{\sum_{k=1}^{m} \sum_{i=1}^{n}\left(\left(\mathscr{T}_{\mathscr{G}\left(\check{d}_{k}\right)}\left(\delta_{i}\right)\right)^{4}+\left(\mathscr{J}_{\mathscr{G}\left(\check{d}_{k}\right)}\left(\delta_{i}\right)\right)^{4}\right)}}$

$\delta_{\text {PFHSS }}((\mathscr{F}, \ddot{A}),(\mathscr{G}, \ddot{\mathfrak{B}}))=1$.

Thus, the required result is proved. 
Definition 10. Let $(\mathscr{F}, \cdots \ddot{\mathscr{A}})=\left\{\left(\delta_{i}, \mathscr{T}_{\mathscr{F}\left(\check{d}_{k}\right)}\left(\delta_{i}\right), \mathscr{J}_{\mathscr{F}\left(\check{d}_{k}\right)}\left(\delta_{i}\right)\right) \mid\right.$ $\left.\delta_{i} \in \mathscr{U}\right\}$ and $(\mathscr{G}, \ddot{B})=\left\{\left(\delta_{i}, \mathscr{T}_{\mathscr{G}\left(\check{d}_{k}\right)}\left(\delta_{i}\right), \mathscr{J}_{\mathscr{G}\left(\check{d}_{k}\right)}\left(\delta_{i}\right)\right) \mid \delta_{i} \in \mathcal{U}\right\}$ be two PFHSSs. Then, their correlation coefficient is given as $\delta_{\text {PFHSS }}((\mathscr{F}, \ddot{\mathscr{A}}),(\mathscr{G}, \ddot{\mathfrak{B}}))$ and is defined as follows:

$$
\begin{aligned}
& \delta_{\mathrm{PFHSS}}^{1}((\mathscr{F}, \cdots \ddot{\mathscr{A}}),(\mathscr{G}, \ddot{\mathfrak{B}}))=\frac{\mathscr{C}_{\mathrm{PFHSS}}((\mathscr{F}, \ddot{\mathscr{A}}),(\mathscr{G}, \ddot{B}))}{\max \left\{\varsigma_{\mathrm{PFHSS}}(\mathscr{F}, \ddot{\mathscr{A}}), \varsigma_{\mathrm{PFHSS}}(\mathscr{G}, \ddot{\mathfrak{B}})\right\}} \\
& \delta_{\mathrm{PFHSS}}^{1}((\mathscr{F}, \ddot{\mathscr{A}}),(\mathscr{G}, \ddot{\mathfrak{B}}))=\frac{\sum_{k=1}^{m} \sum_{i=1}^{n}\left(\left(\mathscr{T}_{\mathscr{F}\left(\check{d}_{k}\right)}\left(\delta_{i}\right)\right)^{2} *\left(\mathscr{T}_{\mathscr{G}\left(\check{d}_{k}\right)}\left(\delta_{i}\right)\right)^{2}+\left(\mathscr{F}_{\mathscr{F}\left(\check{d}_{k}\right)}\left(\delta_{i}\right)\right)^{2} *\left(\mathscr{J}_{\mathscr{G}\left(\check{d}_{k}\right)}\left(\delta_{i}\right)\right)^{2}\right)}{\max \left\{\sum_{k=1}^{m} \sum_{i=1}^{n}\left(\left(\mathscr{T}_{\mathscr{F}\left(\check{d}_{k}\right)}\left(\delta_{i}\right)\right)^{4}+\left(\mathscr{F}_{\mathscr{F}\left(\check{d}_{k}\right)}\left(\delta_{i}\right)\right)^{4}\right), \sum_{k=1}^{m} \sum_{i=1}^{n}\left(\left(\mathscr{T}_{\mathscr{G}\left(\check{d}_{k}\right)}\left(\delta_{i}\right)\right)^{4}+\left(\mathscr{F}_{\mathscr{G}\left(\check{d}_{k}\right)}\left(\delta_{i}\right)\right)^{4}\right)\right\}} .
\end{aligned}
$$

Theorem 3. Let $(\mathscr{F}, \ddot{\mathscr{A}})=\left\{\left(\delta_{i}, \mathscr{T}_{\mathscr{F}\left(\check{d}_{k}\right)}\left(\delta_{i}\right), \mathscr{J}_{\mathscr{F}\left(\check{d}_{k}\right)}\left(\delta_{i}\right)\right) \mid\right.$ $\left.\delta_{i} \in \mathscr{U}\right\}$ and $(\mathscr{G}, \dddot{\mathfrak{B}})=\left\{\left(\delta_{i}, \mathscr{T}_{\mathscr{G}\left(\check{d}_{k}\right)}\left(\delta_{i}\right), \mathscr{J}_{\mathscr{G}\left(\check{d}_{k}\right)}\left(\delta_{i}\right)\right) \mid \delta_{i} \in \mathcal{U}\right\}$ be two PFHSSs. Then, the CC between them satisfies the following properties:

(1) $0 \leq \delta_{\text {PFHSS }}^{1}((\mathscr{F}, \mathscr{A}),(\mathscr{G}, \mathfrak{B})) \leq 1$.

(2) $\delta_{\text {PFHSS }}^{1}((\mathscr{F}, \ddot{\mathscr{A}}),(\mathscr{G}, \dddot{\mathfrak{B}}))=\delta_{\text {PFHSS }}^{1}((\mathscr{G}, \ddot{\mathfrak{B}}),(\mathscr{F}, \ddot{\mathscr{A}}))$.

(3) If $(\mathscr{F}, \ddot{\mathscr{A}})=(\mathscr{G}, \ddot{\mathfrak{B}})$, that is, $\forall i, k, \mathscr{T}_{\mathscr{F}\left(\check{d}_{k}\right)}\left(\delta_{i}\right)=$ $\mathscr{T}_{\mathscr{G}\left(\breve{d}_{k}\right)}\left(\delta_{i}\right)$ and $\mathscr{J}_{\mathscr{F}\left(\check{d}_{k}\right)}\left(\delta_{i}\right)=\mathscr{J}_{\mathscr{G}\left(\check{d}_{k}\right)}\left(\delta_{i}\right)$, then $\delta_{\text {PFHSS }}^{1}((\mathscr{F}, \mathscr{A}),(\mathscr{G}, \ddot{\mathfrak{B}}))=1$.

Proof. It is similar to Theorem 2.

In this era, it is very necessary to consider the weights of PFHSS in practical applications. Whenever the decision- maker adjusts different weights for each alternative in the universe of discourse, the decision may be different. Consequently, it is particularly significant to plan the weight before decision-making. Let $\Omega=\left\{\Omega_{1}, \Omega_{2}, \Omega_{3}, \ldots, \Omega_{m}\right\}^{T}$ be a weight vector for experts such as $\Omega_{k}>0, \sum_{k=1}^{m} \Omega_{k}=1$, and $\gamma=\left\{\gamma_{1}, \gamma_{2}, \gamma_{3}, \ldots, \gamma_{n}\right\}^{T}$ be a weight vector for parameters such as $\gamma_{i}>0, \sum_{i=1}^{n} \gamma_{i}=1$. In the following, we develop the WCC between PFHSS by extending Definitions 9 and 10 .

Definition 11. Let $(\mathscr{F}, \cdots \cdot \mathscr{A})=\left\{\left(\delta_{i}, \mathscr{T}_{\mathscr{F}\left(\check{d}_{k}\right)}\left(\delta_{i}\right), \mathscr{J}_{\mathscr{F}\left(\check{d}_{k}\right)}\left(\delta_{i}\right)\right) \mid\right.$ $\left.\delta_{i} \in \mathscr{U}\right\}$ and $(\mathscr{G}, \ddot{B})=\left\{\left(\delta_{i}, \mathscr{T}_{\mathscr{G}\left(\check{d}_{k}\right)}\left(\delta_{i}\right), \mathscr{J}_{\mathscr{G}\left(\check{d}_{k}\right)}\left(\delta_{i}\right)\right) \mid \delta_{i} \in \mathscr{U}\right\}$ be two PFHSSs. Then, their weighted correlation coefficient is given as $\delta_{\text {WPFHSS }}((\mathscr{F}, \ddot{\mathscr{A}}),(\mathscr{G}, \mathfrak{B}))$ and is defined as follows:

$$
\begin{aligned}
& \delta_{\mathrm{WPFHSS}}((\mathscr{F}, \ddot{\mathscr{A}}),(\mathscr{G}, \mathfrak{B}))=\frac{\mathscr{C}_{\mathrm{WPFHSS}}((\mathscr{F}, \ddot{\mathscr{A}}),(\mathscr{G}, \ddot{\mathfrak{B}}))}{\sqrt{\varsigma_{\mathrm{WPFHSS}}(\mathscr{F}, \ddot{A})} * \sqrt{\varsigma_{\mathrm{WPFHSS}}(\mathscr{G}, \mathfrak{B})}}
\end{aligned}
$$

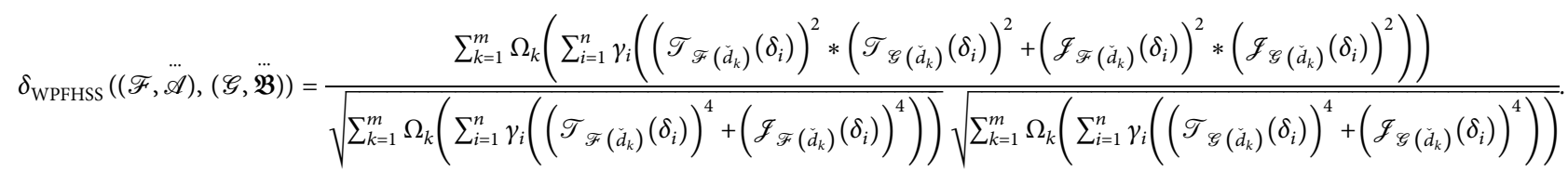


Definition 12. Let $(\mathscr{F}, \cdots \ddot{\mathscr{A}})=\left\{\left(\delta_{i}, \mathscr{T}_{\mathscr{F}\left(\check{d}_{k}\right)}\left(\delta_{i}\right), \mathscr{J}_{\mathscr{F}\left(\check{d}_{k}\right)}\left(\delta_{i}\right)\right) \mid\right.$ $\left.\delta_{i} \in \mathscr{U}\right\}$ and $(\mathscr{G}, \ddot{B})=\left\{\left(\delta_{i}, \mathscr{T}_{\mathscr{G}\left(\check{d}_{k}\right)}\left(\delta_{i}\right), \mathscr{J}_{\mathscr{G}\left(\check{d}_{k}\right)}\left(\delta_{i}\right)\right) \mid \delta_{i} \in \mathcal{U}\right\}$ be two PFHSSs. Then, their weighted correlation coefficient is given as $\delta_{\mathrm{WPFHSS}}^{1}((\mathscr{F}, \cdots \mathscr{A}),(\mathscr{G}, \mathfrak{B}))$ and is defined as follows:

$$
\begin{aligned}
& \delta_{\text {WPFHSS }}^{1}((\mathscr{F}, \ddot{\mathscr{A}}),(\mathscr{G}, \ddot{\mathfrak{B}}))=\frac{\mathscr{C}_{\mathrm{WPFHSS}}((\mathscr{F}, \ddot{A}),(\mathscr{G}, \ddot{B}))}{\max \left\{\varsigma_{\mathrm{WPFHSS}}(\mathscr{F}, \ddot{\mathscr{A}}), \varsigma_{\mathrm{WPFHSS}}(\mathscr{G}, \mathfrak{B})\right\}}
\end{aligned}
$$

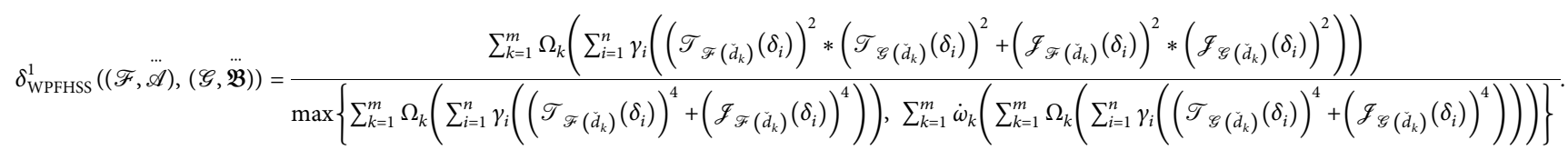

If we consider $\Omega=\{(1 / m),(1 / m), \ldots,(1 / m)\}$ and $\gamma$ $=\{(1 / n),(1 / n), \ldots,(1 / n)\}$, then $\delta_{\text {WPFHSS }}((\mathscr{F}, \ddot{A}),(\mathscr{G}, \ddot{B}))$ and $\delta_{\mathrm{WPFHSS}}^{1}((\mathscr{F}, \ldots \mathscr{A}),(\mathscr{G}, \ddot{\mathfrak{B}})) \quad$ are reduced to $\delta_{\text {WPFHSS }}((\mathscr{F}, \ddot{A}),(\mathscr{G}, \ddot{B})) \quad$ and $\quad \delta_{\text {WPFHSS }}^{1}((\mathscr{F}, \ddot{\mathscr{A}}),(\mathscr{G}, \ddot{\mathfrak{B}}))$, respectively.

Theorem 4. Let $\quad(\mathscr{F}, \ddot{\mathscr{A}})=\left\{\left(\delta_{i}, \mathscr{T}_{\mathscr{F}\left(\check{d}_{k}\right)}\left(\delta_{i}\right), \mathscr{J}_{\mathscr{F}\left(\check{d}_{k}\right)}\left(\delta_{i}\right)\right) \mid\right.$ $\left.\delta_{i} \in \mathscr{U}\right\}$ and $(\mathscr{G}, \dddot{B})=\left\{\left(\delta_{i}, \mathscr{T}_{\mathscr{G}\left(\check{d}_{k}\right)}\left(\delta_{i}\right), \mathscr{J}_{\mathscr{G}\left(\check{d}_{k}\right)}\left(\delta_{i}\right)\right) \mid \delta_{i} \in \mathcal{U}\right\}$ be two PFHSSs. Then, the CC between them satisfies the following properties:

(1) $0 \leq \delta_{\text {WPFHSS }}((\mathscr{F}, \ddot{\mathscr{A}}),(\mathscr{G}, \dddot{\mathfrak{B}})) \leq 1$.
(2) $\delta_{\text {WPFHSS }}((\mathscr{F}, \ddot{\mathscr{A}}),(\mathscr{G}, \ddot{\mathfrak{B}}))=\delta_{\text {WPFHSS }}((\mathscr{G}, \dddot{\mathfrak{B}})$, $(\mathscr{F}, \mathscr{A}))$.

(3) If $(\mathscr{F}, \ddot{A})=(\mathscr{G}, \ddot{B})$, that is, $\quad \forall i, \quad k$, $\mathscr{T}_{\mathscr{F}\left(\check{d}_{k}\right)}\left(\delta_{i}\right)=\mathscr{T}_{\mathscr{G}\left(\check{d}_{k}\right)}\left(\delta_{i}\right)$, and $\quad \mathscr{J}_{\mathscr{F}\left(\check{d}_{k}\right)}\left(\delta_{i}\right)=$ $\mathscr{J}_{\mathscr{G}\left(\check{d}_{k}\right)}\left(\delta_{i}\right)$, then $\delta_{\text {WPFHSS }}((\mathscr{F}, \ddot{\mathscr{A}}),(\mathscr{G}, \ddot{\mathfrak{B}}))=1$.

Proof. The inequality $\delta_{\text {WPFHSS }}((\mathscr{F}, \ddot{\mathscr{A}}),(\mathscr{G}, \mathfrak{B})) \geq 0$ is trivial, and here, we only need to prove that $\delta_{\text {WPFHS }}((\mathscr{F}, \ddot{A}),(\mathscr{G}, \ddot{B})) \leq 1$.

$$
\text { As we know, }
$$

$$
\begin{aligned}
& \mathscr{C}_{\mathrm{WPFSS}}((\mathscr{F}, \ddot{\mathscr{A}}),(\mathscr{G}, \ddot{\mathfrak{B}}))=\sum_{k=1}^{m} \Omega_{k}\left(\sum_{i=1}^{n} \gamma_{i}\left(\left(\mathscr{T}_{\mathscr{F}\left(\check{d}_{k}\right)}\left(\delta_{i}\right)\right)^{2} *\left(\mathscr{T}_{\mathscr{G}\left(\check{d}_{k}\right)}\left(\delta_{i}\right)\right)^{2}+\left(\mathscr{J}_{\mathscr{F}\left(\check{d}_{k}\right)}\left(\delta_{i}\right)\right)^{2} *\left(\mathscr{F}_{\mathscr{G}\left(\check{d}_{k}\right)}\left(\delta_{i}\right)\right)^{2}\right)\right) \\
& =\sum_{k=1}^{m} \Omega_{k}\left(\gamma_{1}\left(\left(\mathscr{T}_{\mathscr{F}\left(\check{d}_{k}\right)}\left(\delta_{1}\right)\right)^{2} *\left(\mathscr{T}_{\mathscr{G}\left(\check{d}_{k}\right)}\left(\delta_{1}\right)\right)^{2}+\left(\mathscr{F}_{\mathscr{F}\left(\check{d}_{k}\right)}\left(\delta_{1}\right)\right)^{2} *\left(\mathscr{J}_{\mathscr{G}\left(\check{d}_{k}\right)}\left(\delta_{1}\right)\right)^{2}\right)\right) \\
& +\sum_{k=1}^{m} \Omega_{k}\left(\gamma_{2}\left(\left(\mathscr{T}_{\mathscr{F}\left(\check{d}_{k}\right)}\left(\delta_{2}\right)\right)^{2} *\left(\mathscr{T}_{\mathscr{G}\left(\check{d}_{k}\right)}\left(\delta_{2}\right)\right)^{2}+\left(\mathscr{J}_{\mathscr{F}\left(\check{d}_{k}\right)}\left(\delta_{2}\right)\right)^{2} *\left(\mathscr{F}_{\mathscr{G}\left(\check{d}_{k}\right)}\left(\delta_{2}\right)\right)^{2}\right)\right) \\
& \begin{array}{r}
+ \\
\vdots \\
+
\end{array} \\
& \sum_{k=1}^{m} \Omega_{k}\left(\gamma_{n}\left(\left(\mathscr{T}_{\mathscr{F}\left(\check{d}_{k}\right)}\left(\delta_{n}\right)\right)^{2} *\left(\mathscr{T}_{\mathscr{G}\left(\check{d}_{k}\right)}\left(\delta_{n}\right)\right)^{2}+\left(\mathscr{F}_{\mathscr{F}\left(\check{d}_{k}\right)}\left(\delta_{n}\right)\right)^{2} *\left(\mathscr{F}_{\mathscr{G}\left(\check{d}_{k}\right)}\left(\delta_{n}\right)\right)^{2}\right)\right) \\
& =\left\{\begin{array}{c}
\Omega_{1}\left(\gamma_{1}\left(\left(\mathscr{T}_{\mathscr{F}\left(\check{d}_{1}\right)}\left(\delta_{1}\right)\right)^{2} *\left(\mathscr{T}_{\mathscr{G}\left(\check{d}_{1}\right)}\left(\delta_{1}\right)\right)^{2}+\left(\mathscr{J}_{\mathscr{F}\left(\check{d}_{1}\right)}\left(\delta_{1}\right)\right)^{2} *\left(\mathscr{J}_{\mathscr{G}\left(\check{d}_{1}\right)}\left(\delta_{1}\right)\right)^{2}\right)\right)+ \\
\Omega_{2}\left(\gamma_{1}\left(\left(\mathscr{T}_{\mathscr{F}\left(\check{d}_{2}\right)}\left(\delta_{1}\right)\right)^{2} *\left(\mathscr{T}_{\mathscr{G}\left(\check{d}_{2}\right)}\left(\delta_{1}\right)\right)^{2}+\left(\mathscr{F}_{\mathscr{F}\left(\check{d}_{2}\right)}\left(\delta_{1}\right)\right)^{2} *\left(\mathscr{F}_{\mathscr{G}\left(\check{d}_{2}\right)}\left(\delta_{1}\right)\right)^{2}\right)\right)+ \\
\vdots \\
\Omega_{m}\left(\gamma_{1}\left(\left(\mathscr{T}_{\mathscr{F}\left(\check{d}_{m}\right)}\left(\delta_{1}\right)\right)^{2} *\left(\mathscr{T}_{\mathscr{G}\left(\check{d}_{m}\right)}\left(\delta_{1}\right)\right)^{2}+\left(\mathscr{F}_{\mathscr{F}\left(\check{d}_{m}\right)}\left(\delta_{1}\right)\right)^{2} *\left(\mathscr{J}_{\mathscr{G}\left(\check{d}_{m}\right)}\left(\delta_{1}\right)\right)^{2}\right)\right)
\end{array}\right\}
\end{aligned}
$$


$+\left\{\begin{array}{c}\Omega_{1}\left(\gamma_{2}\left(\left(\mathscr{T}_{\mathscr{F}\left(\check{d}_{1}\right)}\left(\delta_{2}\right)\right)^{2} *\left(\mathscr{T}_{\mathscr{G}\left(\check{d}_{1}\right)}\left(\delta_{2}\right)\right)^{2}+\left(\mathscr{F}_{\mathscr{F}\left(\check{d}_{1}\right)}\left(\delta_{2}\right)\right)^{2} *\left(\mathscr{J}_{\mathscr{G}\left(\check{d}_{1}\right)}\left(\delta_{2}\right)\right)^{2}\right)\right)+ \\ \Omega_{2}\left(\gamma_{2}\left(\left(\mathscr{T}_{\mathscr{F}\left(\check{d}_{2}\right)}\left(\delta_{2}\right)\right)^{2} *\left(\mathscr{T}_{\mathscr{G}\left(\check{d}_{2}\right)}\left(\delta_{2}\right)\right)^{2}+\left(\mathscr{J}_{\mathscr{F}\left(\check{d}_{2}\right)}\left(\delta_{2}\right)\right)^{2} *\left(\mathscr{F}_{\mathscr{G}\left(\check{d}_{2}\right)}\left(\delta_{2}\right)\right)^{2}\right)\right)+ \\ \vdots \\ \Omega_{m}\left(\gamma_{2}\left(\left(\mathscr{T}_{\mathscr{F}\left(\check{d}_{m}\right)}\left(\delta_{2}\right)\right)^{2} *\left(\mathscr{T}_{\mathscr{G}\left(\check{d}_{m}\right)}\left(\delta_{2}\right)\right)^{2}+\left(\mathscr{F}_{\mathscr{F}\left(\check{d}_{m}\right)}\left(\delta_{2}\right)\right)^{2} *\left(\mathscr{F}_{\mathscr{G}\left(\check{d}_{m}\right)}\left(\delta_{2}\right)\right)^{2}\right)\right)\end{array}\right\}$

$+$

:

$+$

$\left\{\begin{array}{c}\Omega_{1}\left(\gamma_{n}\left(\left(\mathscr{T}_{\mathscr{F}\left(\check{d}_{1}\right)}\left(\delta_{n}\right)\right)^{2} *\left(\mathscr{T}_{\mathscr{G}\left(\check{d}_{1}\right)}\left(\delta_{n}\right)\right)^{2}+\left(\mathscr{F}_{\mathscr{F}\left(\check{d}_{1}\right)}\left(\delta_{n}\right)\right)^{2} *\left(\mathscr{F}_{\mathscr{G}\left(\check{d}_{1}\right)}\left(\delta_{n}\right)\right)^{2}\right)\right)+ \\ \Omega_{2}\left(\gamma_{n}\left(\left(\mathscr{T}_{\mathscr{F}\left(\check{d}_{2}\right)}\left(\delta_{n}\right)\right)^{2} *\left(\mathscr{T}_{\mathscr{G}\left(\check{d}_{2}\right)}\left(\delta_{n}\right)\right)^{2}+\left(\mathscr{F}_{\mathscr{F}\left(\check{d}_{2}\right)}\left(\delta_{n}\right)\right)^{2} *\left(\mathscr{F}_{\mathscr{G}\left(\check{d}_{2}\right)}\left(\delta_{n}\right)\right)^{2}\right)\right)+ \\ \vdots \\ \Omega_{m}\left(\gamma_{n}\left(\left(\mathscr{T}_{\mathscr{F}\left(\check{d}_{m}\right)}\left(\delta_{n}\right)\right)^{2} *\left(\mathscr{T}_{\mathscr{G}\left(\check{d}_{m}\right)}\left(\delta_{n}\right)\right)^{2}+\left(\mathscr{F}_{\mathscr{F}\left(\check{d}_{m}\right)}\left(\delta_{n}\right)\right)^{2} *\left(\mathscr{J}_{\mathscr{G}\left(\check{d}_{m}\right)}\left(\delta_{n}\right)\right)^{2}\right)\right)\end{array}\right\}$

$=\left\{\begin{array}{c}\Omega_{1}\left(\sqrt{\gamma_{1}}\left(\mathscr{T}_{\mathscr{F}\left(\check{d}_{1}\right)}\left(\delta_{1}\right)\right)^{2} * \sqrt{\gamma_{1}}\left(\mathscr{T}_{\mathscr{G}\left(\check{d}_{1}\right)}\left(\delta_{1}\right)\right)^{2}+\sqrt{\gamma_{1}}\left(\mathscr{F}_{\mathscr{F}\left(\check{d}_{1}\right)}\left(\delta_{1}\right)\right)^{2} * \sqrt{\gamma_{1}}\left(\mathscr{F}_{\mathscr{G}\left(\check{d}_{1}\right)}\left(\delta_{1}\right)\right)^{2}\right)+ \\ \Omega_{2}\left(\sqrt{\gamma_{1}}\left(\mathscr{T}_{\mathscr{F}\left(\check{d}_{2}\right)}\left(\delta_{1}\right)\right)^{2} * \sqrt{\gamma_{1}}\left(\mathscr{T}_{\mathscr{G}\left(\check{d}_{2}\right)}\left(\delta_{1}\right)\right)^{2}+\sqrt{\gamma_{1}}\left(\mathscr{F}_{\mathscr{F}\left(\check{d}_{2}\right)}\left(\delta_{1}\right)\right)^{2} * \sqrt{\gamma_{1}}\left(\mathscr{F}_{\mathscr{G}\left(\check{d}_{2}\right)}\left(\delta_{1}\right)\right)^{2}\right)+ \\ \vdots \\ \Omega_{m}\left(\sqrt{\gamma_{1}}\left(\mathscr{T}_{\mathscr{F}\left(\check{d}_{m}\right)}\left(\delta_{1}\right)\right)^{2} * \sqrt{\gamma_{1}}\left(\mathscr{T}_{\mathscr{G}\left(\check{d}_{m}\right)}\left(\delta_{1}\right)\right)^{2}+\sqrt{\gamma_{1}}\left(\mathscr{F}_{\mathscr{F}\left(\check{d}_{m}\right)}\left(\delta_{1}\right)\right)^{2} * \sqrt{\gamma_{1}}\left(\mathscr{J}_{\mathscr{G}\left(\check{d}_{m}\right)}\left(\delta_{1}\right)\right)^{2}\right)\end{array}\right\}$

$+\left\{\begin{array}{c}\Omega_{1}\left(\sqrt{\gamma_{2}}\left(\mathscr{T}_{\mathscr{F}\left(\check{d}_{1}\right)}\left(\delta_{2}\right)\right)^{2} * \sqrt{\gamma_{2}}\left(\mathscr{T}_{\mathscr{G}\left(\check{d}_{1}\right)}\left(\delta_{2}\right)\right)^{2}+\sqrt{\gamma_{2}}\left(\mathscr{J}_{\mathscr{F}\left(\check{d}_{1}\right)}\left(\delta_{2}\right)\right)^{2} * \sqrt{\gamma_{2}}\left(\mathscr{J}_{\mathscr{G}\left(\check{d}_{1}\right)}\left(\delta_{2}\right)\right)^{2}\right)+ \\ \Omega_{2}\left(\sqrt{\gamma_{2}}\left(\mathscr{T}_{\mathscr{F}\left(\check{d}_{2}\right)}\left(\delta_{2}\right)\right)^{2} * \sqrt{\gamma_{2}}\left(\mathscr{T}_{\mathscr{G}\left(\check{d}_{2}\right)}\left(\delta_{2}\right)\right)^{2}+\sqrt{\gamma_{2}}\left(\mathscr{J}_{\mathscr{F}\left(\check{d}_{2}\right)}\left(\delta_{2}\right)\right)^{2} * \sqrt{\gamma_{2}}\left(\mathscr{J}_{\mathscr{G}\left(\check{d}_{2}\right)}\left(\delta_{2}\right)\right)^{2}\right)+ \\ \vdots \\ + \\ \Omega_{m}\left(\sqrt{\gamma_{2}}\left(\mathscr{T}_{\mathscr{F}\left(\check{d}_{m}\right)}\left(\delta_{2}\right)\right)^{2} * \sqrt{\gamma_{2}}\left(\mathscr{T}_{\mathscr{G}\left(\check{d}_{m}\right)}\left(\delta_{2}\right)\right)^{2}+\sqrt{\gamma_{2}}\left(\mathscr{J}_{\mathscr{F}\left(\check{d}_{m}\right)}\left(\delta_{2}\right)\right)^{2} * \sqrt{\gamma_{2}}\left(\mathscr{J}_{\mathscr{G}\left(\check{d}_{m}\right)}\left(\delta_{2}\right)\right)^{2}\right)\end{array}\right\}$ 


$$
\begin{aligned}
& + \\
& \Omega_{1}\left(\sqrt{\gamma_{n}}\left(\mathscr{T}_{\mathscr{F}\left(\check{d}_{1}\right)}\left(\delta_{n}\right)\right)^{2} * \sqrt{\gamma_{n}}\left(\mathscr{T}_{\mathscr{G}\left(\check{d}_{1}\right)}\left(\delta_{n}\right)\right)^{2}+\sqrt{\gamma_{n}}\left(\mathscr{J}_{\mathscr{F}\left(\check{d}_{1}\right)}\left(\delta_{n}\right)\right)^{2} * \sqrt{\gamma_{n}}\left(\mathscr{F}_{\mathscr{G}\left(\check{d}_{1}\right)}\left(\delta_{n}\right)\right)^{2}\right)+ \\
& \Omega_{2}\left(\sqrt{\gamma_{n}}\left(\mathscr{T}_{\mathscr{F}\left(\check{d}_{2}\right)}\left(\delta_{n}\right)\right)^{2} * \sqrt{\gamma_{n}}\left(\mathscr{T}_{\mathscr{G}\left(\check{d}_{2}\right)}\left(\delta_{n}\right)\right)^{2}+\sqrt{\gamma_{n}}\left(\mathscr{J}_{\mathscr{F}\left(\check{d}_{2}\right)}\left(\delta_{n}\right)\right)^{2} * \sqrt{\gamma_{n}}\left(\mathscr{J}_{\mathscr{G}\left(\check{d}_{2}\right)}\left(\delta_{n}\right)\right)^{2}\right)+ \\
& \left.\Omega_{m}\left(\sqrt{\gamma_{n}}\left(\mathscr{T}_{\mathscr{F}\left(\check{d}_{m}\right)}\left(\delta_{n}\right)\right)^{2} * \sqrt{\gamma_{n}}\left(\mathscr{T}_{\mathscr{G}\left(\check{d}_{m}\right)}\left(\delta_{n}\right)\right)^{2}+\sqrt{\gamma_{n}}\left(\mathscr{F}_{\mathscr{F}\left(\check{d}_{m}\right)}\left(\delta_{n}\right)\right)^{2} * \sqrt{\gamma_{n}}\left(\mathscr{F}_{\mathscr{G}\left(\check{d}_{m}\right)}\left(\delta_{n}\right)\right)^{2}\right)\right) \\
& =\left\{\begin{array}{c}
\left(\sqrt{\Omega_{1}} \sqrt{\gamma_{1}}\left(\mathscr{T}_{\mathscr{F}\left(\check{d}_{1}\right)}\left(\delta_{1}\right)\right)^{2} * \sqrt{\Omega_{1}} \sqrt{\gamma_{1}}\left(\mathscr{T}_{\mathscr{G}\left(\check{d}_{1}\right)}\left(\delta_{1}\right)\right)^{2}+\sqrt{\Omega_{1}} \sqrt{\gamma_{1}}\left(\mathscr{F}_{\mathscr{F}\left(\check{d}_{1}\right)}\left(\delta_{1}\right)\right)^{2} * \sqrt{\Omega_{1}} \sqrt{\gamma_{1}}\left(\mathscr{J}_{\mathscr{G}\left(\check{d}_{1}\right)}\left(\delta_{1}\right)\right)^{2}\right)^{2} \\
\left(\sqrt{\Omega_{2}} \sqrt{\gamma_{1}}\left(\mathscr{T}_{\mathscr{F}\left(\check{d}_{2}\right)}\left(\delta_{1}\right)\right)^{2} * \sqrt{\Omega_{2}} \sqrt{\gamma_{1}}\left(\mathscr{T}_{\mathscr{G}\left(\check{d}_{2}\right)}\left(\delta_{1}\right)\right)^{2}+\sqrt{\Omega_{2}} \sqrt{\gamma_{1}}\left(\mathscr{F}_{\mathscr{F}\left(\check{d}_{2}\right)}\left(\delta_{1}\right)\right)^{2} * \sqrt{\Omega_{2}} \sqrt{\gamma_{1}}\left(\mathscr{J}_{\mathscr{G}\left(\check{d}_{2}\right)}\left(\delta_{1}\right)\right)^{2}\right)^{2} \\
\vdots \\
+ \\
\left(\sqrt{\Omega_{m}} \sqrt{\gamma_{1}}\left(\mathscr{T}_{\mathscr{F}\left(\check{d}_{m}\right)}\left(\delta_{1}\right)\right)^{2} * \sqrt{\Omega_{m}} \sqrt{\gamma_{1}}\left(\mathscr{T}_{\mathscr{G}\left(\check{(}_{m}\right)}\left(\delta_{1}\right)\right)^{2}+\sqrt{\Omega_{m}} \sqrt{\gamma_{1}}\left(\mathscr{F}_{\mathscr{F}\left(\check{d}_{m}\right)}\left(\delta_{1}\right)\right)^{2} * \sqrt{\Omega_{m}} \sqrt{\gamma_{1}}\left(\mathscr{J}_{\mathscr{G}\left(\check{d}_{m}\right)}\left(\delta_{1}\right)\right)^{2}\right)
\end{array}\right\} \\
& +\left\{\begin{array}{c}
\left(\sqrt{\Omega_{1}} \sqrt{\gamma_{2}}\left(\mathscr{T}_{\mathscr{F}\left(\check{d}_{1}\right)}\left(\delta_{2}\right)\right)^{2} * \sqrt{\Omega_{1}} \sqrt{\gamma_{2}}\left(\mathscr{T}_{\mathscr{G}\left(\check{d}_{1}\right)}\left(\delta_{2}\right)\right)^{2}+\sqrt{\Omega_{1}} \sqrt{\gamma_{2}}\left(\mathscr{J}_{\mathscr{F}\left(\check{d}_{1}\right)}\left(\delta_{2}\right)\right)^{2} * \sqrt{\Omega_{1}} \sqrt{\gamma_{2}}\left(\mathscr{J}_{\mathscr{G}\left(\check{d}_{1}\right)}\left(\delta_{2}\right)\right)^{2}\right)+ \\
\left(\sqrt{\Omega_{2}} \sqrt{\gamma_{2}}\left(\mathscr{T}_{\mathscr{F}\left(\check{d}_{2}\right)}\left(\delta_{2}\right)\right)^{2} * \sqrt{\Omega_{2}} \sqrt{\gamma_{2}}\left(\mathscr{T}_{\mathscr{G}\left(\check{d}_{2}\right)}\left(\delta_{2}\right)\right)^{2}+\sqrt{\Omega_{2}} \sqrt{\gamma_{2}}\left(\mathscr{F}_{\mathscr{F}\left(\check{d}_{2}\right)}\left(\delta_{2}\right)\right)^{2} * \sqrt{\Omega_{2}} \sqrt{\gamma_{2}}\left(\mathscr{F}_{\mathscr{G}\left(\check{d}_{2}\right)}\left(\delta_{2}\right)\right)^{2}\right)+ \\
\vdots \\
+ \\
\left(\sqrt{\Omega_{m}} \sqrt{\gamma_{2}}\left(\mathscr{T}_{\mathscr{F}\left(\check{d}_{m}\right)}\left(\delta_{2}\right)\right)^{2} * \sqrt{\Omega_{m}} \sqrt{\gamma_{2}}\left(\mathscr{T}_{\mathscr{G}\left(\check{d}_{m}\right)}\left(\delta_{2}\right)\right)^{2}+\sqrt{\Omega_{m}} \sqrt{\gamma_{2}}\left(\mathscr{J}_{\mathscr{F}\left(\check{d}_{m}\right)}\left(\delta_{2}\right)\right)^{2} * \sqrt{\Omega_{m}} \sqrt{\gamma_{2}}\left(\mathscr{J}_{\mathscr{G}\left(\check{d}_{m}\right)}\left(\delta_{2}\right)\right)^{2}\right)
\end{array}\right\} \\
& + \\
& \vdots \\
& \left\{\begin{array}{c}
\left(\sqrt{\Omega_{1}} \sqrt{\gamma_{n}}\left(\mathscr{T}_{\mathscr{F}\left(\check{d}_{1}\right)}\left(\delta_{n}\right)\right)^{2} * \sqrt{\Omega_{1}} \sqrt{\gamma_{n}}\left(\mathscr{T}_{\mathscr{G}\left(\check{d}_{1}\right)}\left(\delta_{n}\right)\right)^{2}+\sqrt{\Omega_{1}} \sqrt{\gamma_{n}}\left(\mathscr{F}_{\mathscr{F}\left(\check{d}_{1}\right)}\left(\delta_{n}\right)\right)^{2} * \sqrt{\Omega_{1}} \sqrt{\gamma_{n}}\left(\mathscr{J}_{\mathscr{G}\left(\check{d}_{1}\right)}\left(\delta_{n}\right)\right)^{2}\right)^{2} \\
\left(\sqrt{\Omega_{2}} \sqrt{\gamma_{n}}\left(\mathscr{T}_{\mathscr{F}\left(\check{d}_{2}\right)}\left(\delta_{n}\right)\right)^{2} * \sqrt{\Omega_{2}} \sqrt{\gamma_{n}}\left(\mathscr{T}_{\mathscr{G}\left(\check{d}_{2}\right)}\left(\delta_{n}\right)\right)^{2}+\sqrt{\Omega_{2}} \sqrt{\gamma_{n}}\left(\mathscr{J}_{\mathscr{F}\left(\check{d}_{2}\right)}\left(\delta_{n}\right)\right)^{2} * \sqrt{\Omega_{2}} \sqrt{\gamma_{n}}\left(\mathscr{J}_{\mathscr{G}\left(\check{d}_{2}\right)}\left(\delta_{n}\right)\right)^{2}\right)^{+} \\
\vdots \\
+ \\
\left(\sqrt{\Omega_{m}} \sqrt{\gamma_{n}}\left(\mathscr{T}_{\mathscr{F}\left(\check{d}_{m}\right)}\left(\delta_{n}\right)\right)^{2} * \sqrt{\Omega_{m}} \sqrt{\gamma_{n}}\left(\mathscr{T}_{\mathscr{G}\left(\check{d}_{m}\right)}\left(\delta_{n}\right)\right)^{2}+\sqrt{\Omega_{m}} \sqrt{\gamma_{n}}\left(\mathscr{J}_{\mathscr{F}\left(\check{d}_{m}\right)}\left(\delta_{n}\right)\right)^{2} * \sqrt{\Omega_{m}} \sqrt{\gamma_{n}}\left(\mathscr{J}_{\mathscr{G}\left(\check{d}_{m}\right)}\left(\delta_{n}\right)\right)^{2}\right)
\end{array}\right\} .
\end{aligned}
$$


By using Cauchy-Schwarz inequality, we get

$\mathscr{C}_{\text {WPFHSS }}((\mathscr{F}, \ddot{\mathscr{A}}),(\mathscr{G}, \mathfrak{B}))^{2}$

$$
\begin{aligned}
& \leq\left\{\begin{array}{c}
\left\{\begin{array}{c}
\Omega_{1} \gamma_{1}\left(\left(\mathscr{T}_{\mathscr{F}\left(\check{d}_{1}\right)}\left(\delta_{1}\right)\right)^{4}+\left(\mathscr{J}_{\mathscr{F}\left(\check{d}_{1}\right)}\left(\delta_{1}\right)\right)^{4}\right)+\Omega_{2} \gamma_{1}\left(\left(\mathscr{T}_{\mathscr{F}\left(\check{d}_{2}\right)}\left(\delta_{1}\right)\right)^{4}+\left(\mathscr{F}_{\mathscr{F}\left(\check{d}_{2}\right)}\left(\delta_{1}\right)\right)^{4}\right)+\cdots+ \\
\Omega_{m} \gamma_{1}\left(\left(\mathscr{T}_{\mathscr{F}\left(\check{d}_{m}\right)}\left(\delta_{1}\right)\right)^{4}+\left(\mathscr{J}_{\mathscr{F}\left(\check{d}_{m}\right)}\left(\delta_{1}\right)\right)^{4}\right)
\end{array}\right\}+ \\
\left\{\begin{array}{c}
\Omega_{1} \gamma_{2}\left(\left(\mathscr{T}_{\mathscr{F}\left(\check{d}_{1}\right)}\left(\delta_{2}\right)\right)^{4}+\left(\mathscr{F}_{\mathscr{F}\left(\check{d}_{1}\right)}\left(\delta_{2}\right)\right)^{4}\right)+\Omega_{2} \gamma_{2}\left(\left(\mathscr{T}_{\mathscr{F}\left(\check{d}_{2}\right)}\left(\delta_{2}\right)\right)^{4}+\left(\mathscr{F}_{\mathscr{F}\left(\check{d}_{2}\right)}\left(\delta_{2}\right)\right)^{4}\right)+\cdots+ \\
\Omega_{m} \gamma_{m}\left(\left(\mathscr{T}_{\mathscr{F}\left(\check{d}_{m}\right)}\left(\delta_{2}\right)\right)^{4}+\left(\mathscr{F}_{\mathscr{F}\left(\check{d}_{m}\right)}\left(\delta_{2}\right)\right)^{4}\right) \\
\vdots \\
+
\end{array}\right\}+ \\
\left\{\begin{array}{c}
\Omega_{1} \gamma_{n}\left(\left(\mathscr{T}_{\mathscr{F}\left(\check{d}_{1}\right)}\left(\delta_{n}\right)\right)^{4}+\left(\mathscr{F}_{\mathscr{F}\left(\check{d}_{1}\right)}\left(\delta_{n}\right)\right)^{4}\right)+\Omega_{2} \gamma_{n}\left(\left(\mathscr{T}_{\mathscr{F}\left(\check{d}_{2}\right)}\left(\delta_{n}\right)\right)^{4}+\left(\mathscr{F}_{\mathscr{F}\left(\check{d}_{2}\right)}\left(\delta_{n}\right)\right)^{4}\right)+\cdots+ \\
\Omega_{m} \gamma_{n}\left(\left(\mathscr{T}_{\mathscr{F}\left(\check{d}_{m}\right)}\left(\delta_{n}\right)\right)^{4}+\left(\mathscr{F}_{\mathscr{F}\left(\check{d}_{m}\right)}\left(\delta_{n}\right)\right)^{4}\right)
\end{array}\right.
\end{array}\right\} \\
& \cdot\left\{\begin{array}{c}
\left\{\begin{array}{c}
\Omega_{1} \gamma_{1}\left(\left(\mathscr{T}_{\mathscr{G}\left(\check{d}_{1}\right)}\left(\delta_{1}\right)\right)^{4}+\left(\mathscr{F}_{\mathscr{G}\left(\check{d}_{1}\right)}\left(\delta_{1}\right)\right)^{4}\right)+\Omega_{2} \gamma_{1}\left(\left(\mathscr{T}_{\mathscr{G}\left(\check{d}_{2}\right)}\left(\delta_{1}\right)\right)^{4}+\left(\mathscr{F}_{\mathscr{G}\left(\check{d}_{2}\right)}\left(\delta_{1}\right)\right)^{4}\right)+\cdots+ \\
\Omega_{m} \gamma_{1}\left(\left(\mathscr{T}_{\mathscr{G}\left(\check{d}_{m}\right)}\left(\delta_{1}\right)\right)^{4}+\left(\mathscr{J}_{\mathscr{G}\left(\check{d}_{m}\right)}\left(\delta_{1}\right)\right)^{4}\right)
\end{array}\right\}+ \\
\left\{\begin{array}{c}
\Omega_{1} \gamma_{2}\left(\left(\mathscr{T}_{\mathscr{G}\left(\check{d}_{1}\right)}\left(\delta_{2}\right)\right)^{4}+\left(\mathscr{J}_{\mathscr{G}\left(\check{d}_{1}\right)}\left(\delta_{2}\right)\right)^{4}\right)+\Omega_{2} \gamma_{2}\left(\left(\mathscr{T}_{\mathscr{G}\left(\check{d}_{2}\right)}\left(\delta_{2}\right)\right)^{4}+\left(\mathscr{J}_{\mathscr{G}\left(\check{d}_{2}\right)}\left(\delta_{2}\right)\right)^{4}\right)+\cdots+ \\
\Omega_{m} \gamma_{m}\left(\left(\mathscr{T}_{\mathscr{G}\left(\check{d}_{m}\right)}\left(\delta_{2}\right)\right)^{4}+\left(\mathscr{F}_{\mathscr{G}\left(\check{d}_{m}\right)}\left(\delta_{2}\right)\right)^{4}\right) \\
\vdots \\
+
\end{array}\right\}+ \\
\left\{\begin{array}{c}
\Omega_{1} \gamma_{n}\left(\left(\mathscr{T}_{\mathscr{G}\left(\check{d}_{1}\right)}\left(\delta_{n}\right)\right)^{4}+\left(\mathscr{J}_{\mathscr{G}\left(\check{d}_{1}\right)}\left(\delta_{n}\right)\right)^{4}\right)+\Omega_{2} \gamma_{n}\left(\left(\mathscr{T}_{\mathscr{G}\left(\check{d}_{2}\right)}\left(\delta_{n}\right)\right)^{4}+\left(\mathscr{J}_{\mathscr{G}\left(\check{d}_{2}\right)}\left(\delta_{n}\right)\right)^{4}\right)+\cdots+ \\
\Omega_{m} \gamma_{n}\left(\left(\mathscr{T}_{\mathscr{G}\left(\check{d}_{m}\right)}\left(\delta_{n}\right)\right)^{4}+\left(\mathscr{F}_{\mathscr{G}\left(\check{d}_{m}\right)}\left(\delta_{n}\right)\right)^{4}\right)
\end{array}\right.
\end{array}\right\}
\end{aligned}
$$

$$
\mathscr{C}_{\text {WPFHSS }}((\mathscr{F}, \ddot{A}),(\mathscr{G}, \mathfrak{B}))^{2}
$$$$
\leq \sum_{k=1}^{m} \Omega_{k}\left(\sum_{i=1}^{n} \gamma_{i}\left(\left(\mathscr{T}_{\mathscr{F}\left(\check{d}_{k}\right)}\left(\delta_{i}\right)\right)^{4}+\left(\mathscr{F}_{\mathscr{F}\left(\check{d}_{k}\right)}\left(\delta_{i}\right)\right)^{4}\right)\right) \times \sum_{k=1}^{m} \Omega_{k}\left(\sum_{i=1}^{n} \gamma_{i}\left(\left(\mathscr{T}_{\mathscr{G}\left(\check{d}_{k}\right)}\left(\delta_{i}\right)\right)^{4}+\left(\mathscr{J}_{\mathscr{G}\left(\check{d}_{k}\right)}\left(\delta_{i}\right)\right)^{4}\right)\right)
$$

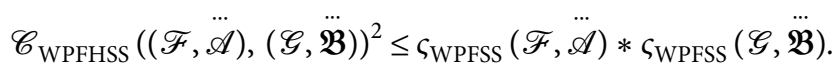

Therefore, $\quad \mathscr{C}_{\text {WPFHSS }}((\mathscr{F}, \mathscr{A}), \quad(\mathscr{G}, \mathfrak{B})) \leq \quad$ Proof. 2 2. The proof is obvious.

$\sqrt{\varsigma_{\mathrm{WPFSS}}(\mathscr{F}, \ddot{\mathscr{A}}) * \varsigma_{\mathrm{WPFSS}}(\mathscr{G}, \mathfrak{B})}$; hence, $0 \leq \delta_{\mathrm{WPFSS}}((\mathscr{F}, \ddot{\mathscr{A}})$, $(\mathscr{G}, \mathfrak{B})) \leq 1$.

Proof. 3. From Equation (25), we have

$\delta_{\mathrm{WPFHSS}}((\mathscr{F}, \ddot{\mathscr{A}}),(\mathscr{G}, \ddot{\mathfrak{B}}))=\frac{\sum_{k=1}^{m} \Omega_{k}\left(\sum_{i=1}^{n} \gamma_{i}\left(\left(\mathscr{T}_{\mathscr{F}\left(\check{d}_{k}\right)}\left(\delta_{i}\right)\right)^{2} *\left(\mathscr{T}_{\mathscr{G}\left(\check{d}_{k}\right)}\left(\delta_{i}\right)\right)^{2}+\left(\mathscr{F}_{\mathscr{F}\left(\check{d}_{k}\right)}\left(\delta_{i}\right)\right)^{2} *\left(\mathscr{F}_{\mathscr{G}\left(\check{d}_{k}\right)}\left(\delta_{i}\right)\right)^{2}\right)\right)}{\sqrt{\sum_{k=1}^{m} \Omega_{k}\left(\sum_{i=1}^{n} \gamma_{i}\left(\left(\mathscr{T}_{\mathscr{F}\left(\check{d}_{k}\right)}\left(\delta_{i}\right)\right)^{4}+\left(\mathscr{F}_{\mathscr{F}\left(\tilde{d}_{k}\right)}\left(\delta_{i}\right)\right)^{4}\right)\right)} \sqrt{\sum_{k=1}^{m} \Omega_{k}\left(\sum_{i=1}^{n} \gamma_{i}\left(\left(\mathscr{T}_{\mathscr{G}\left(\check{d}_{k}\right)}\left(\delta_{i}\right)\right)^{4}+\left(\mathscr{J}_{\mathscr{G}\left(\check{d}_{k}\right)}\left(\delta_{i}\right)\right)^{4}\right)\right)}}$. 
As we know that $\mathscr{T}_{\mathscr{F}\left(d_{k}\right)}\left(\delta_{i}\right)=\mathscr{T}_{\mathscr{G}\left(\check{d}_{k}\right)}\left(\delta_{i}\right)$ and $\mathscr{J}_{\mathscr{F}\left(\check{d}_{k}\right)}\left(\delta_{i}\right)=\mathscr{J}_{\mathscr{G}\left(\check{d}_{k}\right)}\left(\delta_{i}\right) \forall i, k$, we get

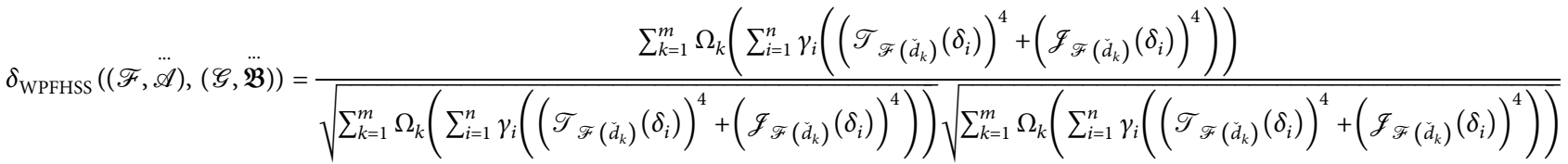

$\delta_{\text {WPFHSS }}((\mathscr{F}, \dddot{\mathscr{A}}),(\mathscr{G}, \dddot{\mathfrak{B}}))=1$.

Thus, the required result is proved.

Definition 13. Let $\mathfrak{J}_{\check{d}_{k}}=\left(\mathscr{T}_{\check{d}_{k}}, \mathscr{J}_{\check{d}_{k}}\right), \quad \widetilde{J}_{\check{d}_{11}}=\left(\mathscr{T}_{\check{d}_{11}}, \mathscr{J}_{\check{d}_{11}}\right)$, and $\mathfrak{\Im}_{\check{d}_{12}}=\left(\mathscr{T}_{\check{d}_{12}}, \mathscr{J}_{\check{d}_{12}}\right)$ be three PFHSNs and $\alpha$ be a positive real number; by algebraic norms, we have

(1) $\widetilde{J}_{\check{d}_{11}} \oplus \widetilde{J}_{\check{d}_{12}}=\left\langle\sqrt{\mathscr{T}_{\breve{d}_{11}}^{2}+\mathscr{T}_{\breve{d}_{12}}^{2}-\mathscr{T}_{\breve{d}_{11}}^{2} \mathscr{T}_{\breve{d}_{12}}^{2}}, \mathscr{J}_{\check{d}_{11}} \mathscr{J}_{\check{d}_{12}}\right\rangle$.

(2) $\widetilde{J}_{\check{d}_{11}} \otimes \widetilde{J}_{\check{d}_{12}}=\left\langle\mathscr{T}_{\check{d}_{11}} \mathscr{T}_{\check{d}_{12}}, \sqrt{\mathscr{J}_{\check{d}_{11}}^{2}+\mathscr{J}_{\check{d}_{12}}^{2}-\mathscr{J}_{\check{d}_{11}}^{2} \mathscr{J}_{\check{d}_{12}}^{2}}\right\rangle$.

(3) $\alpha \widetilde{\mathfrak{J}}_{\check{d}_{k}}=\left\langle\sqrt{1-\left(1-\mathscr{T}_{\breve{d}_{k}}^{2}\right)^{\alpha}}, \mathscr{J}_{\breve{d}_{k}}^{\alpha}\right\rangle$.

(4) $\mathfrak{J}_{\tilde{d}_{k}}^{\alpha}=\left\langle\mathscr{T}_{\tilde{d}_{k}}^{\alpha}, \sqrt{1-\left(1-\mathcal{J}_{\breve{d}_{k}}^{2}\right)^{\alpha}}\right\rangle$.
Some averaging and geometric aggregation operators for PFHSSs have been defined based on the above laws for the collection of PFHSNs.

Definition 14. Let $\mathfrak{\Im}_{\check{d}_{i j}}=\left(\mathscr{T}_{\check{d}_{i j}}, \mathscr{J}_{\check{d}_{i j}}\right)$ be a PFHSN, $\Omega_{i}$ and $\gamma_{j}$ be the weight vector for expert's and subattributes of the considered parameters, respectively, with given conditions $\Omega_{i}>0, \sum_{i=1}^{n} \Omega_{i}=1, \gamma_{j}>0$, and $\sum_{j=1}^{m} \gamma_{j}=1$. Then, PFHSWA operator is defined as PFHSWA: $\Delta^{n} \longrightarrow \Delta$, which is defined as follows:

$$
\begin{aligned}
\operatorname{PFHSWA}\left(\mathfrak{\Im}_{\check{d}_{11}}, \mathfrak{J}_{\check{d}_{12}}, \ldots, \mathfrak{J}_{\check{d}_{n m}}\right) & =\oplus_{j=1}^{m} \gamma_{j}\left(\oplus_{i=1}^{n} \Omega_{i} \mathfrak{\Im}_{\check{d}_{i j}}\right) \\
& =\left\langle\sqrt{1-\prod_{j=1}^{m}\left(\prod_{i=1}^{n}\left(1-\mathscr{T}_{\check{d}_{i j}}^{2}\right)^{\Omega_{i}}\right)^{\gamma_{j}}}, \prod_{j=1}^{m}\left(\prod_{i=1}^{n}\left(\mathscr{J}_{\check{d}_{i j}}\right)^{\Omega_{i}}\right)^{\gamma_{j}}\right\rangle,
\end{aligned}
$$

where $\Omega_{i}$ and $\gamma_{j}$ are the weight vector for experts and subattributes of the parameters, respectively, with given conditions $\Omega_{i}>0, \sum_{i=1}^{n} \Omega_{i}=1, \gamma_{j}>0$, and $\sum_{j=1}^{m} \gamma_{j}=1$.

\section{Remark 2.}

(1) If both $\mathscr{T}_{\breve{d}_{i j}}^{2}+\mathscr{J}_{\breve{d}_{i j}}^{2} \leq 1$ and $\mathscr{T}_{\breve{d}_{i j}}+\mathscr{J}_{\breve{d}_{i j}} \leq 1$ hold, then the PFHSWA operator was reduced to the IFHSWA operator [56].

(2) If both $\mathscr{T}_{\check{d}_{i j}}^{2}+\mathscr{J}_{\check{d}_{i j}}^{2} \leq 1$ and $\mathscr{T}_{\check{d}_{i j}}+\mathscr{J}_{\check{d}_{i j}} \leq 1$ hold and a set of attributes contains only one parameter with no subattributes, then the PFHSWA operator was reduced to the IFSWA operator [59].

Definition 15. Let $\mathfrak{J}_{\check{d}_{i j}}=\left(\mathscr{T}_{\check{d}_{i j}}, \mathscr{J}_{\check{d}_{i j}}\right)$ be a PFHSN, and $\Omega_{i}$ and $\gamma_{j}$ be the weight vector for experts and subattributes of considered parameters, respectively, with given conditions $\Omega_{i}>0, \sum_{i=1}^{n} \Omega_{i}=1, \gamma_{j}>0$, and $\sum_{j=1}^{m} \gamma_{j}=1$, then PFHSWG operator is defined as PFHSWG: $\Delta^{n} \longrightarrow \Delta$, which is defined as follows:

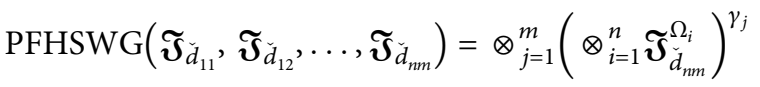

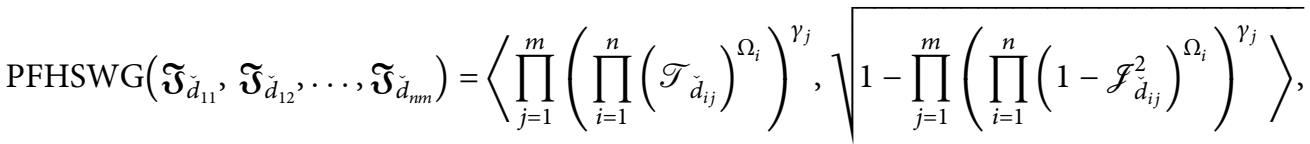


where $\Omega_{i}$ and $\gamma_{j}$ are the weight vector for experts and subattributes of the given parameters, respectively, with given conditions $\Omega_{i}>0, \sum_{i=1}^{n} \Omega_{i}=1, \gamma_{j}>0$, and $\sum_{j=1}^{m} \gamma_{j}=1$.

\section{Remark 3.}

(1) If both $\mathscr{T}_{\breve{d}_{i j}}^{2}+\mathscr{J}_{\breve{d}_{i j}}^{2} \leq 1$ and $\mathscr{T}_{\check{d}_{i j}}+\mathscr{J}_{\check{d}_{i j}} \leq 1$ hold, then the PFHSWG operator was reduced to the IFHSWG operator [56].

(2) If both $\mathscr{T}_{\breve{d}_{i j}}^{2}+\mathscr{J}_{\breve{d}_{i j}}^{2} \leq 1$ and $\mathscr{T}_{\breve{d}_{i j}}+\mathscr{J}_{\breve{d}_{i j}} \leq 1$ hold and a set of attributes contains only one parameter with no subattributes, then the PFHSWG operator was reduced to the IFSWG operator [59].

\section{TOPSIS Approach on PFHSS for MAGDM Problem Based on the Correlation Coefficient}

In this section, we are going to develop a methodology for solving decision-making issues through continuing the TOPSIS method for PFHSS information based on correlation coefficients. Hwang and Yoon [37] developed the TOPSIS method and used it to promote the order of evaluation components of positive and negative ideal solutions for decision-making issues. By utilizing the TOPSIS method, we will be able to discover the best possible choices which have the smallest and largest distances to PIS and NIS, respectively. The TOPSIS technique ensures that the correlation measure can be used to distinguish positive ideals from negative ideals by choosing rankings. Generally, investigators are using the TOPSIS method to find closeness coefficients along with distinctive distance forms as well as comparable measures. The TOPSIS technique with correlation coefficients is superior for locating closeness coefficients instead of distance as well as similarity measures because the correlation measure retains the linear relationship between the factors considered. By utilizing the developed CC, a TOPSIS method is presented to select the most suitable option.

4.1. Proposed Decision-Making Approach. Assume a set of " $s$ " alternatives such as $Q=\left\{Q^{1}, Q^{2}, Q^{3}, \ldots, Q^{s}\right\}$ for assessment under the team of experts $\mathscr{X}=\left\{\mathscr{X}_{1}, \mathscr{X}_{2}, \mathscr{X}_{3}, \ldots, \mathscr{X}_{n}\right\}$ with weights $\Omega=\left(\Omega_{1}, \Omega_{1}, \ldots, \Omega_{n}\right)^{T}$ and $\Omega_{i}>0, \sum_{i=1}^{n} \Omega_{i}=1$. Let $\mathbb{Q}=\left\{d_{1}, d_{2}, \ldots, d_{m}\right\}$ be a set of attributes and $\mathfrak{J}^{\prime}=\left\{\left(d_{1 \rho} \times d_{2 \rho} \times \cdots \times d_{m \rho}\right)\right.$, for all $\left.\rho \in\{1,2, \ldots, t\}\right\}$, be a collection of their corresponding subattributes, with weights $\gamma=\left(\gamma_{1 \rho}, \gamma_{2 \rho}, \gamma_{3 \rho}, \ldots, \gamma_{m \rho}\right)^{T}$ such as $\gamma_{\rho}>0$, $\sum_{\rho=1}^{t} \gamma_{\rho}=1$. The elements in the collection of subattributes are multivalued; for the sake of convenience, the elements of $\mathfrak{\Im}^{\prime}$ can be expressed as $\mathfrak{\Im}^{\prime}=\left\{\check{d}_{\partial}: \partial \in\{1,2, \ldots, k\}\right\}$. The team of experts $\left\{\mathscr{X}_{i}: i=1,2, \ldots, n\right\}$ evaluate the alternatives $\left\{Q^{(z)}: z=1,2, \ldots, s\right\}$ based on the desired subattributes of the considered parameters $\left\{\check{d}_{\partial}: \partial=1,2, \ldots, k\right\}$ given in the form of PFHSNs such as $\left(\mathfrak{J}_{\tilde{d}_{i j}}^{(z)}\right)_{n \times \partial}=\left(\mathscr{T}_{\check{d}_{i j}}^{(z)}, \mathscr{J}_{\breve{d}_{i j}}^{(z)}\right)_{n \times \partial}$, where $0 \leq \mathscr{T}_{\tilde{d}_{i j}}^{(z)}, \mathscr{J}_{d_{i j}}^{(z)} \leq 1$, and $\left(\mathscr{T}_{d_{i j}}^{(z)}\right)^{2}+\left(\mathscr{J}_{\tilde{d}_{i j}}^{(z)}\right)^{2} \leq 1$ for all $i, j$.

Step 1. Construct a matrix for each alternative $\left\{Q^{(z)}: z=1,2, \ldots, s\right\}$ in the form of PFHSNs by using subattributes of the given attributes such as follows:

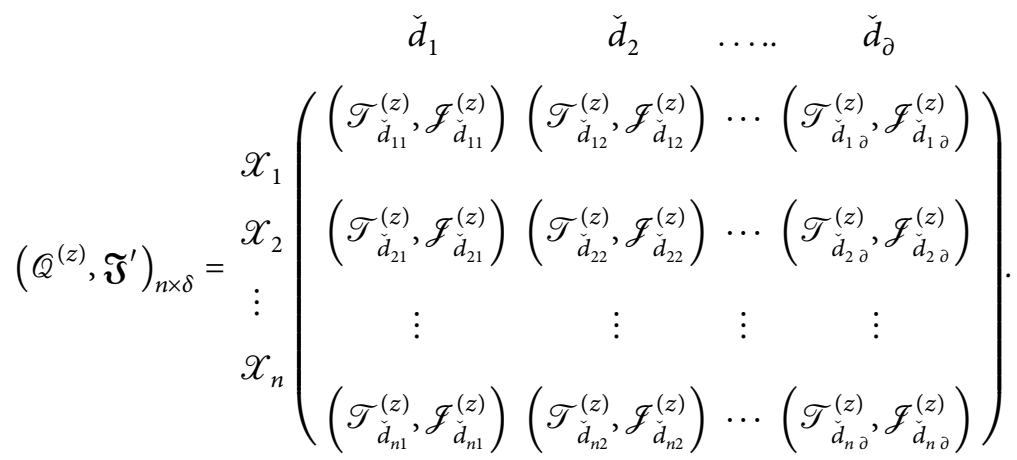

Step 2. Normalize the collective information decision matrix by converting the rating value of the cost-type parameters into benefit-type parameters with the help of normalization formula:

$$
h_{i j}= \begin{cases}\mathfrak{J}_{\check{d}_{i j}}^{c}=\left(\mathscr{J}_{\check{d}_{i j}}^{(z)}, \mathscr{T}_{\check{d}_{i j}}^{(z)}\right) ; & \text { cost }- \text { type parameter, } \\ \mathfrak{J}_{\check{d}_{i j}}=\left(\mathscr{T}_{\check{d}_{i j}}^{(z)}, \mathscr{J}_{\check{d}_{i j}}^{(z)}\right) ; & \text { benefit }- \text { type parameter. }\end{cases}
$$

Step 3. Construct the weighted decision matrix for each alternative $\overline{\mathbb{Q}}^{(z)}=\left(\overline{\mathfrak{J}}_{\tilde{d}_{i j}}^{(z)}\right)_{n \times \partial}$, where

$$
\begin{aligned}
\overline{\mathfrak{J}}_{\mathfrak{d}_{i j}}^{(z)} & =\gamma_{j} \Omega_{i} \mathfrak{J}_{d_{i j}}^{(z)}=\left(\sqrt{1-\left(\left(1-\mathscr{T}_{\mathfrak{d}_{i j}}^{2}\right)^{\Omega_{i}}\right)^{\gamma_{j}}},\left(\left(\mathscr{J}_{\check{d}_{i j}}\right)^{\Omega_{i}}\right)^{\gamma_{j}}\right) \\
& =\left(\overline{\mathscr{T}}_{\dot{d}_{i j}}^{(z)}, \overline{\mathscr{J}}_{\dot{d}_{i j}}^{(z)}\right),
\end{aligned}
$$

where $\Omega_{i}$ and $\gamma_{j}$ are the weights for the $i^{\text {th }}$ expert and $j^{\text {th }}$ subattribute, respectively. 
Step 4. Find the indices $h_{i j}=\operatorname{argmax}_{z}\left\{\theta_{i j}^{(z)}\right\}$ and $g_{i j}=\operatorname{argmin}_{z}\left\{\theta_{i j}^{(z)}\right\}$ for each expert $\mathscr{X}_{i}$ and subattribute $\check{d}_{j}$ from CC matrices and determine the PIA and NIA based on indices as follows:

$$
\mathscr{L}^{+}=\left(\mathscr{T}_{d_{i j}}^{+}, \mathscr{F}_{d_{i j}}^{+}\right)_{n \times \partial}=\left(\overline{\mathscr{T}}_{\tilde{d}_{i j}}^{\left(h_{i j}\right)}, \overline{\mathscr{J}}_{\hat{d}_{i j}}^{\left(h_{i j}\right)}\right),
$$

$$
\mathscr{L}^{-}=\left(\mathcal{T}_{\tilde{d}_{i j}}^{-}, \mathscr{F}_{\dot{d}_{i j}}^{-}\right)_{n \times \partial}=\left(\overline{\mathscr{T}}_{\check{d}_{i j}}^{\left(g_{i j}\right)}, \overline{\mathcal{J}}_{\dot{d}_{i j}}^{\left(g_{i j}\right)}\right) .
$$

Step 5. Compute the CC between each alternative of weighted decision matrices $\overline{\mathbb{Q}}^{(z)}$ and PIA $\mathscr{L}^{+}$as follows:

$$
\begin{aligned}
p^{(z)} & =\delta_{\text {PFHSS }}\left(\overline{\mathscr{Q}}^{(z)}, \mathscr{L}^{+}\right)=\frac{\mathscr{C}_{\mathrm{PFHSS}}\left(\overline{\mathscr{Q}}^{(z)}, \mathscr{L}^{+}\right)}{\sqrt{\varsigma_{\mathrm{PFHSS}} \widehat{Q}^{(z)} * \varsigma_{\mathrm{PFHSS}} \mathscr{L}^{+}}} \\
& =\frac{\sum_{j=1}^{m} \sum_{i=1}^{n}\left(\overline{\mathscr{T}}_{d_{i j}}^{(z)} * \mathscr{T}_{d_{i j}}^{+}+\overline{\mathscr{J}}_{d_{i j}}^{(z)} * \mathscr{J}_{d_{i j}}^{+}\right)}{\sqrt{\sum_{j=1}^{m} \sum_{i=1}^{n}\left(\left(\overline{\mathscr{T}}_{d_{i j}}^{(z)}\right)^{2}+\left(\overline{\mathscr{F}}_{d_{i j}}^{(z)}\right)^{2}\right)} \sqrt{\sum_{j=1}^{m} \sum_{i=1}^{n}\left(\left(\mathscr{T}_{d_{i j}}^{+}\right)^{2}+\left(\mathscr{F}_{d_{i j}}^{+}\right)^{2}\right)}} .
\end{aligned}
$$

Step 6. Compute the CC between each alternative of the weighted decision matrix $\bar{Q}^{(z)}$ and NIA $\mathscr{L}^{-}$as follows:

$$
\begin{aligned}
& q^{(z)}=\delta_{\mathrm{PFHSS}}\left(\overline{\mathscr{Q}}^{(z)}, \mathscr{L}^{-}\right)=\frac{\mathscr{C}_{\mathrm{PFHSS}}\left(\overline{\bar{Q}}^{(z)}, \mathscr{L}^{-}\right)}{\sqrt{\varsigma_{\mathrm{PFHSS}} \overline{\mathscr{Q}}^{(z)} * \varsigma_{\mathrm{PFHSS}} \mathscr{L}^{-}}} \\
& =\frac{\sum_{j=1}^{m} \sum_{i=1}^{n}\left(\overline{\mathscr{T}}_{d_{i j}}^{(z)} * \mathscr{T}_{\bar{d}_{i j}}^{-}+\overline{\mathscr{J}}_{\dot{d}_{i j}}^{(z)} * \mathscr{J}_{\dot{d}_{i j}}^{-}\right)}{\sqrt{\sum_{j=1}^{m} \sum_{i=1}^{n}\left(\left(\overline{\mathscr{T}}_{d_{i j}}^{(z)}\right)^{2}+\left(\overline{\mathscr{J}}_{d_{i j}}^{(z)}\right)^{2}\right)} \sqrt{\sum_{j=1}^{m} \sum_{i=1}^{n}\left(\left(\mathscr{T}_{\check{d}_{i j}}^{-}\right)^{2}+\left(\mathscr{F}_{\check{d}_{i j}}^{-}\right)^{2}\right)}} .
\end{aligned}
$$

Step 7. The closeness coefficient for each alternative can be found as follows:

$$
\mathscr{R}^{(z)}=\frac{\mathscr{K}\left(\overline{\mathbb{Q}}^{(z)}, \mathscr{L}^{-}\right)}{\mathscr{K}\left(\overline{\mathbb{Q}}^{(z)}, \mathscr{L}^{+}\right)+\mathscr{K}\left(\overline{\mathbb{Q}}^{(z)}, \mathscr{L}^{-}\right)},
$$

where

$$
\begin{aligned}
& \mathscr{K}\left(\overline{\mathbb{Q}}^{(z)}, \mathscr{L}^{-}\right)=1-q^{(z)}, \\
& \mathscr{K}\left(\overline{\bar{Q}}^{(z)}, \mathscr{L}^{+}\right)=1-p^{(z)} .
\end{aligned}
$$

Step 8. Choose the alternative with a maximum value of closeness coefficient.

Step 9. Analyze the ranking of the alternatives.

The flow chart of the proposed method can be seen in Figure 1.

4.2. Selection of an Effective Mask Based on the Proposed TOPSIS Technique. To show the importance as well as the usefulness of the proposed model based on the PFHSS data, we investigated the numerical example of choosing an antivirus mask in the global serious situation of the COVID-19 disease. Everybody in the world has quite a lot of trouble in acquiring an excellent as well as multipurpose antivirus mask to prevent themselves from contracting COVID-19. In this risky COVID-19 situation, the overall demand for antivirus masks seems to have exaggerated. Due to increased demand, it is really difficult to find suitable gas masks in the market. The increase in demand has also caused second-class gas masks to come into the market. Raza et al. [60] discussed the complex transmission of the epidemic problems by utilizing the nonlinear fractional-order Ebola virus mathematical model. They developed the fractional-order Ebola virus transmission model for the treatment and control to reduce its effect on a population that plays an important role in public health. Ahmed et al. [61] introduced some models for the COVID-19 that can address important questions about global health care and suggest important notes. Yang et al. [57] and Shahzadi and Akram [58] used the spherical normal fuzzy sets and formation fuzzy soft Yager ordered weighted average and geometric operators for the selection of effective gas masks during the COVID-19 pandemic. The major inspiration of the current application is to pick out multipurpose gas masks according to the TOPSIS technique for PFHSS to reduce the 


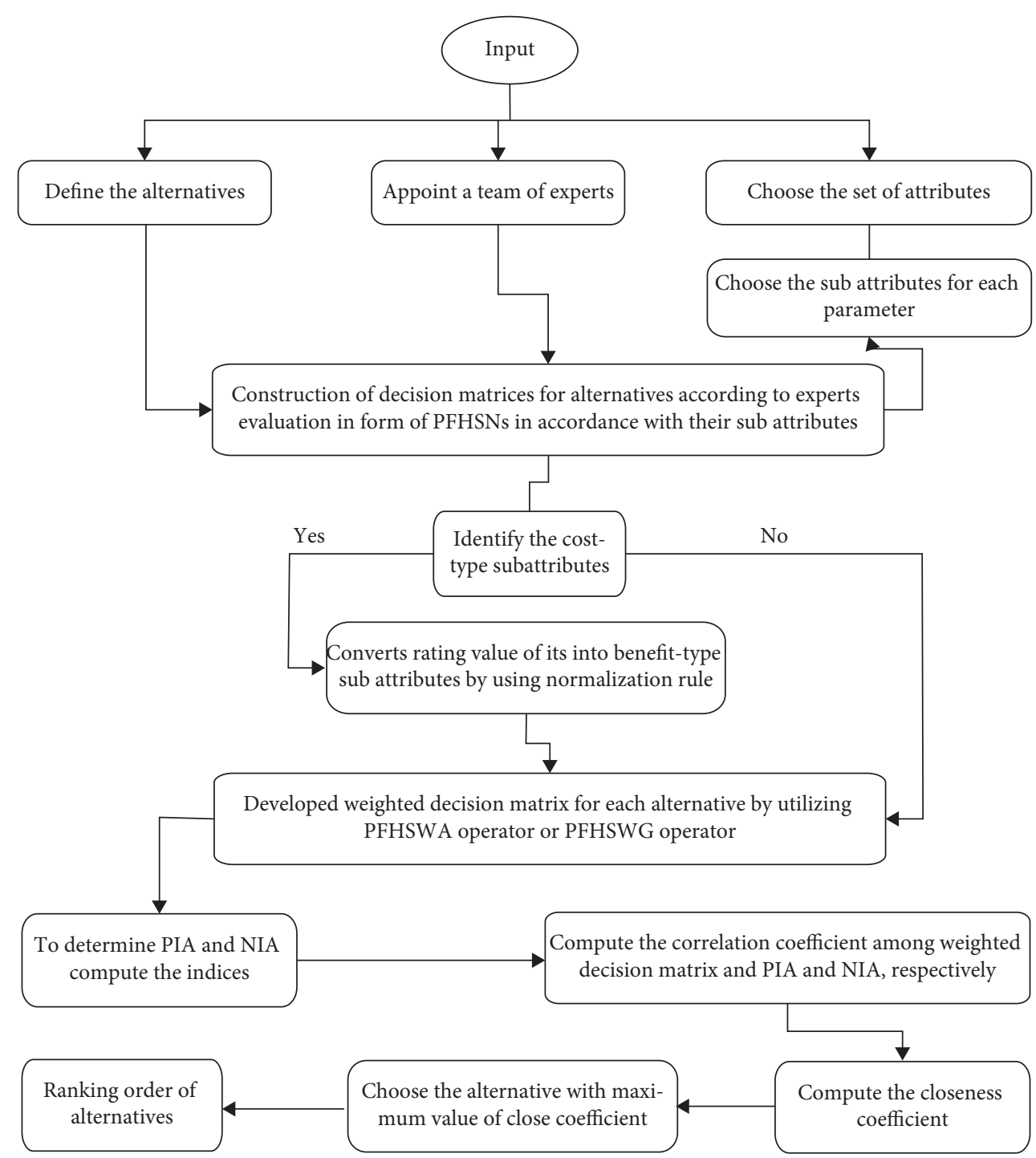

FIgURE 1: Flow chart of TOPSIS method under PFHSS Scenario.

spreading of coronavirus. The team of four experts $\mathscr{X}=\left\{\mathscr{X}_{1}\right.$, $\left.X_{2}, X_{3}, X_{4}\right\}$ having weights $(0.2,0.3,0.4,0.1)^{T}$ evaluated the rating of the different types of antivirus masks $Q=\left\{Q^{1}=\right.$ KN95, $\mathbb{Q}^{2}=$ disposable medical mask, $\mathbb{Q}^{3}=$ surgical mask, $\mathbb{Q}^{4}$ $=$ home - made cloth mask $\}$. The team of experts decides the criteria (attributes) for the selection of antivirus mask as follows: let $\mathscr{L}=\left\{\ell_{1}=\right.$ anti - static properties, $\ell_{2}=$ efficiency, $\ell_{3}=$ quality $\}$ be a collection of attributes, and their corresponding subattributes are given as antistatic properties $=\ell_{1}=$ $\left\{d_{11}=\right.$ limited performance of protective clothingagainst the flame spread $d_{12}=$ Protection against infectious bacteria\}, efficie ncy $=\ell_{2}=\left\{d_{21}=\right.$ Good breathability, $d_{22}=$ Good filtering res ults $\}$, and quality $=\ell_{3}=\left\{d_{31}=\right.$ leakage ratio, $d_{32}=$ reusability $\}$. Let $\mathfrak{J}^{\prime}=\ell_{1} \times \ell_{2} \times \ell_{3}$ be a set of subattributes:

$$
\begin{aligned}
\mathfrak{J}^{\prime} & =\ell_{1} \times \ell_{2} \times \ell_{3}=\left\{d_{11}, d_{12}\right\} \times\left\{d_{21}, d_{22}\right\} \times\left\{d_{31}, d_{32}\right\} \\
& =\left\{\begin{array}{l}
\left(d_{11}, d_{21}, d_{31}\right),\left(d_{11}, d_{21}, d_{32}\right),\left(d_{11}, d_{22}, d_{31}\right),\left(d_{11}, d_{22}, d_{32}\right), \\
\left(d_{12}, d_{21}, d_{31}\right),\left(d_{12}, d_{21}, d_{32}\right),\left(d_{12}, d_{22}, d_{31}\right),\left(d_{12}, d_{22}, d_{32}\right)
\end{array}\right\} .
\end{aligned}
$$

Let $\mathfrak{\Im}^{\prime}=\left\{\check{d}_{1}, \check{d}_{2}, \check{d}_{3}, \check{d}_{4}, \check{d}_{5}, \check{d}_{6}, \check{d}_{7}, \check{d}_{8}\right\}$ be a set of all multisubattributes with weights $(0.12,0.18,0.1,0.15,0.05,0.22$, $0.08,0.1)^{T}$. Each expert will evaluate the ratings of antivirus masks in the form of PFHSNs for each subattribute of the considered parameters. The developed method to find the best alternative is as follows (Tables 1-4):
(1) Step 1. Develop the decision matrices for each alternative under defined multi-subattributes according to each decision-makers rating in terms of PFHSNs.

(2) Step 2. All criteria are of benefit types, so no need to normalize them. 
TABLE 1: Decision matrix for alternative $\mathbb{Q}^{(1)}$.

\begin{tabular}{lcccccccc}
\hline $\mathscr{Q}^{(1)}$ & $\check{\mathbf{d}}_{1}$ & $\check{\mathbf{d}}_{2}$ & $\check{\mathbf{d}}_{3}$ & $\check{\mathbf{d}}_{4}$ & $\check{\mathbf{d}}_{5}$ & $\check{\mathbf{d}}_{6}$ & $\check{\mathbf{d}}_{7}$ & $\check{\mathbf{d}}_{8}$ \\
\hline $\mathscr{X}_{1}$ & $(0.3,0.8)$ & $(0.7,0.3)$ & $(0.6,0.7)$ & $(0.5,0.4)$ & $(0.2,0.4)$ & $(0.4,0.6)$ & $(0.5,0.8)$ & $(0.9,0.3)$ \\
$\mathscr{X}_{2}$ & $(0.6,0.7)$ & $(0.4,0.6)$ & $(0.3,0.4)$ & $(0.9,0.2)$ & $(0.3,0.8)$ & $(0.2,0.4)$ & $(0.7,0.5)$ & $(0.4,0.5)$ \\
$\mathscr{X}_{3}$ & $(0.7,0.3)$ & $(0.2,0.5)$ & $(0.1,0.6)$ & $(0.3,0.4)$ & $(0.4,0.6)$ & $(0.8,0.4)$ & $(0.6,0.7)$ & $(0.2,0.5)$ \\
$\mathscr{X}_{4}$ & $(0.8,0.4)$ & $(0.2,0.9)$ & $(0.2,0.4)$ & $(0.4,0.6)$ & $(0.6,0.5)$ & $(0.5,0.6)$ & $(0.4,0.5)$ & $(0.8,0.3)$ \\
\hline
\end{tabular}

TABle 2: Decision matrix for alternative $\mathbb{Q}^{(2)}$.

\begin{tabular}{lcccccccc}
\hline $\boldsymbol{Q}^{(2)}$ & $\check{\mathbf{d}}_{1}$ & $\check{\mathbf{d}}_{2}$ & $\check{\mathbf{d}}_{3}$ & $\check{\mathbf{d}}_{4}$ & $\check{\mathbf{d}}_{5}$ & $\check{\mathbf{d}}_{6}$ & $\check{\mathbf{d}}_{7}$ & $\check{\mathbf{d}}_{8}$ \\
\hline $\boldsymbol{X}_{1}$ & $(0.7,0.6)$ & $(0.3,0.4)$ & $(0.6,0.5)$ & $(0.3,0.9)$ & $(0.5,0.4)$ & $(0.4,0.6)$ & $(0.7,0.5)$ & $(0.4,0.8)$ \\
$\mathscr{X}_{2}$ & $(0.8,0.5)$ & $(0.7,0.4)$ & $(0.9,0.2)$ & $(0.7,0.4)$ & $(0.4,0.5)$ & $(0.9,0.3)$ & $(0.2,0.7)$ & $(0.3,0.8)$ \\
$\mathscr{X}_{3}$ & $(0.3,0.7)$ & $(0.4,0.5)$ & $(0.4,0.8)$ & $(0.3,0.4)$ & $(0.6,0.7)$ & $(0.3,0.4)$ & $(0.9,0.2)$ & $(0.7,0.2)$ \\
$\mathscr{X}_{4}$ & $(0.5,0.4)$ & $(0.7,0.6)$ & $(0.9,0.3)$ & $(0.8,0.5)$ & $(0.9,0.2)$ & $(0.2,0.4)$ & $(0.4,0.6)$ & $(0.6,0.5)$ \\
\hline
\end{tabular}

TABle 3: Decision matrix for alternative $\mathbb{Q}^{(3)}$.

\begin{tabular}{lcccccccc}
\hline$\widetilde{Q}^{(3)}$ & $\check{\mathbf{d}}_{1}$ & $\check{\mathbf{d}}_{2}$ & $\check{\mathbf{d}}_{3}$ & $\check{\mathbf{d}}_{4}$ & $\check{\mathbf{d}}_{5}$ & $\check{\mathbf{d}}_{6}$ & $\check{\mathbf{d}}_{7}$ & $\check{\mathbf{d}}_{8}$ \\
\hline $\boldsymbol{X}_{1}$ & $(0.5,0.7)$ & $(0.8,0.5)$ & $(0.7,0.4)$ & $(0.4,0.3)$ & $(0.4,0.9)$ & $(0.2,0.4)$ & $(0.8,0.4)$ & $(0.7,0.5)$ \\
$\mathscr{X}_{2}$ & $(0.8,0.5)$ & $(0.7,0.4)$ & $(0.8,0.5)$ & $(0.5,0.2)$ & $(0.5,0.7)$ & $(0.7,0.5)$ & $(0.7,0.6)$ & $(0.6,0.4)$ \\
$\mathscr{X}_{3}$ & $(0.6,0.8)$ & $(0.4,0.5)$ & $(0.6,0.5)$ & $(0.6,0.4)$ & $(0.7,0.5)$ & $(0.8,0.4)$ & $(0.5,0.8)$ & $(0.4,0.5)$ \\
$\mathscr{X}_{4}$ & $(0.5,0.7)$ & $(0.9,0.3)$ & $(0.3,0.5)$ & $(0.5,0.7)$ & $(0.3,0.5)$ & $(0.8,0.5)$ & $(0.7,0.5)$ & $(0.2,0.5)$ \\
\hline
\end{tabular}

TABle 4: Decision matrix for alternative $Q^{(4)}$.

\begin{tabular}{lcccccccc}
\hline $\boldsymbol{Q}^{(4)}$ & $\check{\mathbf{d}}_{1}$ & $\check{\mathbf{d}}_{2}$ & $\check{\mathbf{d}}_{3}$ & $\check{\mathbf{d}}_{4}$ & $\check{\mathbf{d}}_{5}$ & $\check{\mathbf{d}}_{6}$ & $\check{\mathbf{d}}_{7}$ & $\check{\mathbf{d}}_{8}$ \\
\hline $\boldsymbol{X}_{1}$ & $(0.5,0.7)$ & $(0.8,0.5)$ & $(0.7,0.4)$ & $(0.4,0.3)$ & $(0.4,0.9)$ & $(0.2,0.4)$ & $(0.8,0.4)$ & $(0.7,0.5)$ \\
$\mathscr{X}_{2}$ & $(0.8,0.5)$ & $(0.7,0.4)$ & $(0.8,0.5)$ & $(0.5,0.2)$ & $(0.5,0.7)$ & $(0.7,0.5)$ & $(0.7,0.6)$ & $(0.6,0.4)$ \\
$\mathscr{X}_{3}$ & $(0.5,0.4)$ & $(0.4,0.8)$ & $(0.5,0.6)$ & $(0.3,0.4)$ & $(0.7,0.6)$ & $(0.7,0.5)$ & $(0.4,0.9)$ & $(0.5,0.2)$ \\
$\mathscr{X}_{4}$ & $(0.4,0.7)$ & $(0.1,0.3)$ & $(0.7,0.5)$ & $(0.5,0.8)$ & $(0.3,0.5)$ & $(0.8,0.3)$ & $(0.3,0.5)$ & $(0.2,0.5)$ \\
\hline
\end{tabular}

(3) Step 3. Construct the weighted decision matrix for each alternative $\bar{Q}(z)=\left(\overline{\mathscr{L}}_{i j}(z)\right)_{n \times \partial}$ by using Equation (35) given in Tables 5-8.
(4) Step 4. Determine the PIA and NIA based on indices by using Equations (36) and (37):

$$
\begin{aligned}
\mathscr{L}^{+} & =\left[\begin{array}{lllllllll}
(0.6867,0.9915) & (0.5691,0.9576) & (0.6791,0.9762) & (0.6694,0.9848) & (0.8241,0.9840) & (0.5021,0.9778) & (0.8073,0.9889) & (0.6791,0.9762) \\
(0.7200,0.9872) & (0.6109,0.9517) & (0.7068,0.9529) & (0.7781,0.9301) & (0.9070,0.9821) & (0.6509,0.9553) & (0.8033,0.9915) & (0.7068,0.9933) \\
(0.7443,0.9438) & (0.6422,0.9513) & (0.7270,0.9798) & (0.7386,0.9465) & (0.8527,0.9683) & (0.4959,0.9225) & (0.7749,0.9498) & (0.7819,0.9727) \\
(0.6328,0.9891) & (0.4402,0.9908) & (0.6829,0.9909) & (0.5644,0.9821) & (0.8457,0.9974) & (0.4321,0.9800) & (0.7648,0.9945) & (0.6339,0.9880)
\end{array}\right], \\
\mathscr{L}^{-}= & {\left[\begin{array}{lllllllll}
(0.7559,0.9835) & (0.6572,0.9754) & (0.7920,0.9862) & (0.7048,0.9645) & (0.9028,0.9909) & (0.5987,0.9699) & (0.8491,0.9854) & (0.7920,0.9862) \\
(0.7599,0.9754) & (0.7038,0.9728) & (0.9085,0.9333) & (0.9009,0.9301) & (0.9070,0.9897) & (0.4661,0.9236) & (0.9089,0.9621) & (0.8671,0.9645) \\
(0.8678,0.9257) & (0.7379,0.9513) & (0.9083,0.9529) & (0.8376,0.9465) & (0.9190,0.9898) & (0.6897,0.9408) & (0.8509,0.9781) & (0.9083,0.9377) \\
(0.7229,0.9891) & (0.5034,0.9786) & (0.7408,0.9949) & (0.6667,0.9821) & (0.9995,0.9919) & (0.6575,0.9506) & (0.7866,0.9872) & (0.7631,0.9840)
\end{array}\right] . }
\end{aligned}
$$

(5) Step 5. Compute the CC between $\bar{Q}^{(\mathbf{z})}$ and PIA $\mathscr{L}^{+}$by using Equation (38), given $p^{(1)}=0.99915, p^{(2)}$ $=0.99811, p^{(3)}=0.99746$, and $p^{(4)}=0.99787$.
(6) Step 6. Compute the CC between $\overline{\mathbb{Q}}^{(\mathbf{z})}$ and NIA $\mathscr{L}^{-}$ by using Equation (39), given $q^{(1)}=0.99742, q^{(2)}$ $=0.99806, q^{(3)}=0.99870$, and $q^{(4)}=0.99870$. 


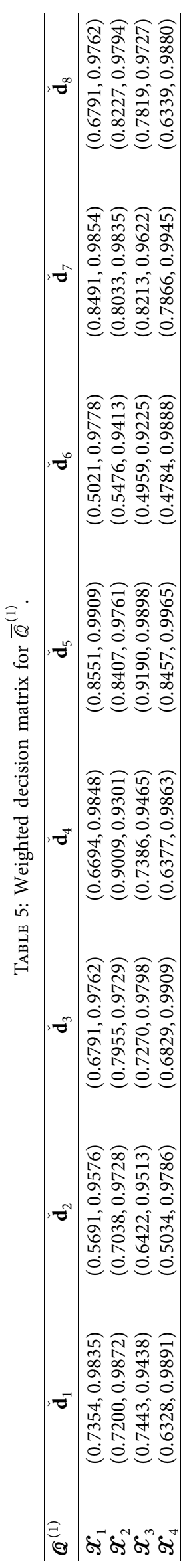




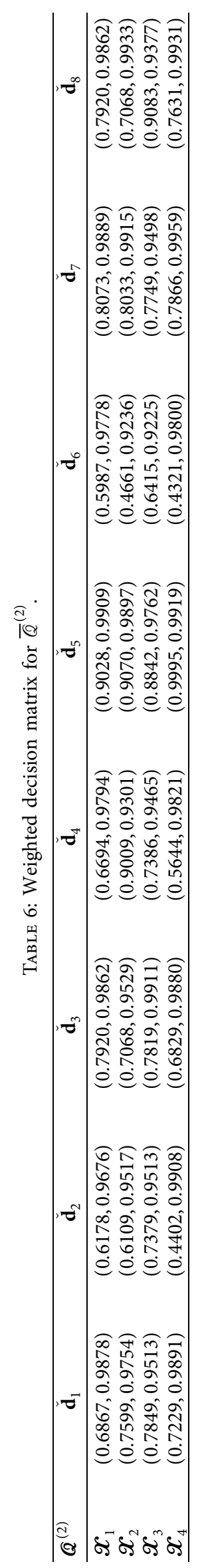




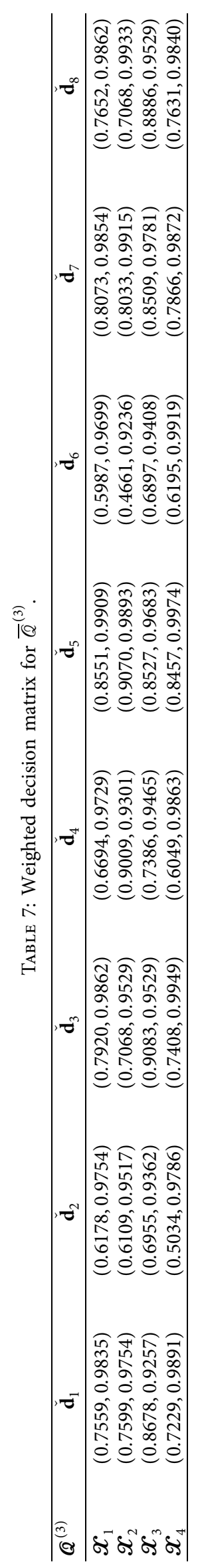




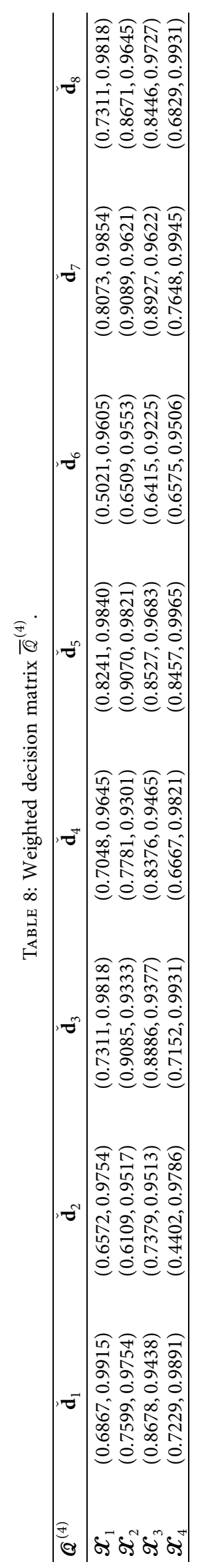




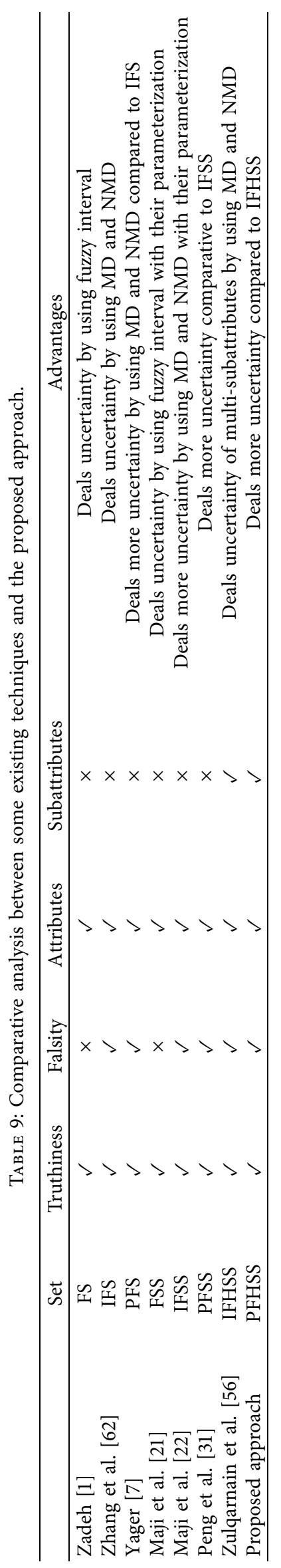


(7) Step 7. Compute the closeness coefficient by using Equation (40), $\mathscr{R}^{(1)}=0.75219, \mathscr{R}^{(2)}=0.50653, \mathscr{R}^{(3)}$ $=0.33854$, and $\mathscr{R}^{(4)}=0.37901$.

(8) Step 8. Choose the alternative with maximum closeness coefficient $\mathscr{R}^{(1)}=0.75219$, so $\mathscr{Q}^{(1)}$ is the best alternative.

(9) Step 9. Analyzing the ranking of the alternatives, we can see $\mathscr{R}^{(1)}>\mathscr{R}^{(2)}>\mathscr{R}^{(4)}>\mathscr{R}^{(3)}$, so the ranking of the alternatives is $\mathscr{Q}^{(1)}>\mathscr{Q}^{(2)}>\mathscr{Q}^{(4)}>\mathscr{Q}^{(3)}$.

\section{Discussion and Comparative Analysis}

In the following sections, we will discuss the effectiveness, naivety, flexibility, and advantages of the proposed method and algorithms. We also organized a brief comparison of the following: the recommended method and available methods.

5.1. Superiority of the Proposed Method. Through this research and comparative analysis, we have concluded that the results of this method are more universal than that of the existing techniques. However, in the decision-making process, compared with the existing decision-making methods, it contains more information to deal with the uncertainty of the data. Besides, many hybrid structures of FSs have become special cases of PFHSSs, and some suitable conditions have been added. Among them, the information related to the object can be expressed more accurately and empirically, so it is a convenient tool to combine inaccurate and uncertain information in the decision-making process. Therefore, our proposed method is effective, flexible, simple, and superior to other hybrid structures of fuzzy sets.

5.2. Comparative Analysis. By using the technique of Zadeh [1], we can process the MD of the attributes, but this method cannot deal with the NMD and subattributes of the considered parameters. Zhang et al. [62] dealt with uncertainty by using $\mathrm{MD}$ and NMD, but these theories also has some limitations such as when the sum of MD and NMD exceeds 1 , then these theories cannot handle the situation. To overcome such difficulties, Yager [7] developed the PFS, but PFS cannot deal with the parametric values of the alternatives. Maji et al. [21] established the FSS to handle the parameterization of the alternatives. The FSS has no information about the NMD of the alternative's attributes, and it deals only with the MD of the attributes. On the contrary, our presented PFHSS handles the uncertainty by utilizing the MD and NMD. Maji et al. [22] proposed the IFSS to accommodate the uncertainty by using MD and NMD of the attributes with their parameterization. The IFSS is unable to solve these problems, where the sum of MD and NMD exceeds one. Peng et al. [31] developed the PFSS to handle the uncertainty competently compared to IFSS. When attributes have their corresponding subattributes, then all the abovementioned theories fail to handle the situation. Zulqarnain et al. [56] presented the TOPSIS technique under the IFHSS environment to deal with uncertain problems by using MD and NMD in which the sum of subattributes of the considered parameters cannot exceed one. When the sum of MD and NMD of subattributes exceeds one such as $\mathscr{T}_{\mathscr{F}(\check{d})}(\delta)+\mathscr{F}_{\mathscr{F}(\check{d})}(\delta) \geq 1$, then IFHSS cannot accommodate the situation. To overcome the above limitations, we extended the IFHSS to PFHSS by modifying the condition $\mathscr{T}_{\mathscr{F}(\check{d})}(\delta)+\mathscr{J}_{\mathscr{F}(\check{d})}(\delta) \leq 1$ to $\left(\mathscr{T}_{\mathscr{F}(\check{d})}(\delta)\right)^{2}+\left(\mathscr{J}_{\mathscr{F}(\check{d})}(\delta)\right)^{2} \leq 1$. Instead, the method we developed is an advanced technique that can handle alternatives with multiple subattribute information listed in Table 9. On the contrary, the established method in this research deals with the uncertainty by using the MD and NMD of the subattributes of alternatives. Therefore, the developed technique is more competent compared to the existing methodologies and surely provides better outcomes for decision-makers during the decisionmaking process.

\section{Conclusion}

The notion of PFHSS is used to solve the problem that contains insufficient information, ambiguity, and inconsistency by considering the degree of membership and nonmembership of the subattributes of considered attributes. Among them, we developed the CC and WCC for PFHSS and demonstrated their desirable characteristics. Similarly, based on the developed correlation, an extended TOPSIS method is introduced by considering the attribute set with its corresponding subattributes and decisionmakers. To find PIA and NIA, we developed correlation indices. The closeness coefficients have been developed based on the established TOPSIS technique to calculate the ranking of alternatives. Also, PFHSWA and PFHSWG operators defined and introduced a decision-making approach based on the developed TOPSIS method. By using the proposed TOPSIS method, a numerical illustration to solve the MAGDM problem has been described. In addition, a comparative analysis was carried out to verify the effectiveness and demonstration of the presented method. Finally, based on the obtained results, it can be concluded that the proposed technique shows higher stability and practicality for decision-makers in the decision-making process. Future research will concentrate on presenting ideas to decision-making complications to several operators in the PFHSS environment. Several other structures, such as topological structures, algebraic structures, and ordered structures, can be developed and investigated under considered environment. This research article has pragmatic boundaries and can be immensely helpful in real-life dimensions, including medical profession, pattern recognition, and economics. We are sure this article will open new vistas for researchers in this field.

\section{Data Availability}

No data were used to support this study.

\section{Conflicts of Interest}

The authors declare that they have no conflicts of interest. 


\section{Acknowledgments}

The author Rifaqat Ali would like to express his gratitude to Deanship of Scientific Research at King Khalid University, Saudi Arabia, for providing funding under the research grant number R.G.P. 2/71/41.

\section{References}

[1] L. A. Zadeh, "Fuzzy sets," Information and Control, vol. 8, no. 3, pp. 338-353, 1965.

[2] K. T. Atanassov, "Intuitionistic fuzzy sets," Fuzzy Sets and Systems, vol. 20, no. 1, pp. 87-96, 1986.

[3] H. Garg and G. Kaur, "Cubic intuitionistic fuzzy sets and its fundamental properties," Journal of Multiple-Valued Logic \& Soft Computing, vol. 33, pp. 507-537, 2019.

[4] K. Atanassov and G. Gargov, "Interval valued intuitionistic fuzzy sets," Fuzzy Sets and Systems, vol. 31, no. 3, pp. 343-349, 1989.

[5] H. Garg and K. Kumar, "Linguistic interval-valued Atanassov intuitionistic fuzzy sets and their applications to group decision making problems," IEEE Transactions on Fuzzy Systems, vol. 27, no. 12, pp. 2302-2311, 2019.

[6] R. R. Yager, "Pythagorean fuzzy subsets," in Procedings Joint IFSA World Congress and NAFIPS Annual Meeting, pp. 57-61, Edmonton, Canada, June 2013.

[7] R. R. Yager, "Pythagorean membership grades in multicriteria decision making," IEEE Transactions on Fuzzy Systems, vol. 22, no. 4, pp. 958-965, 2014.

[8] X. Zhang and Z. Xu, "Extension of TOPSIS to multiple criteria decision making with Pythagorean fuzzy sets," International Journal of Intelligent Systems, vol. 29, no. 12, pp. 1061-1078, 2014.

[9] G. Wei and M. Lu, "Pythagorean fuzzy power aggregation operators in multiple attribute decision making," International Journal of Intelligent Systems, vol. 33, no. 1, pp. 169-186, 2018.

[10] L. Wang and N. Li, "Pythagorean fuzzy interaction power Bonferroni mean aggregation operators in multiple attribute decision making," International Journal of Intelligent Systems, vol. 35, no. 1, pp. 150-183, 2020.

[11] X. Zhang, "A novel approach based on similarity measure for Pythagorean fuzzy multiple criteria group decision making," International Journal of Intelligent Systems, vol. 31, no. 6, pp. 593-611, 2016.

[12] H. Garg, "A new generalized Pythagorean fuzzy information aggregation using Einstein operations and its application to decision making," International Journal of Intelligent Systems, vol. 31, no. 9, pp. 886-920, 2016.

[13] X. Peng and Y. Yang, "Some results for Pythagorean fuzzy sets," International Journal of Intelligent Systems, vol. 30, no. 11, pp. 1133-1160, 2015.

[14] H. Garg, "New logarithmic operational laws and their aggregation operators for Pythagorean fuzzy set and their applications," International Journal of Intelligent Systems, vol. 34, no. 1, p. 82, 2019.

[15] H. Gao, M. Lu, G. Wei, and Y. Wei, "Some novel Pythagorean fuzzy interaction aggregation operators in multiple attribute decision making," Fundamenta Informaticae, vol. 159, no. 4, pp. 385-428, 2018.

[16] X. Peng and H. Yuan, "Fundamental properties of Pythagorean fuzzy aggregation operators," Fundamenta Informaticae, vol. 147, no. 4, pp. 415-446, 2016.
[17] Z. Ma and Z. Xu, "Symmetric Pythagorean fuzzy weighted geometric/averaging operators and their application in multicriteria decision-making problems," International Journal of Intelligent Systems, vol. 31, pp. 1-22, 2016.

[18] D. Molodtsov, "Soft set theory-First results," Computers \& Mathematics with Applications, vol. 37, no. 4-5, pp. 19-31, 1999.

[19] P. K. Maji, R. Biswas, and A. R. Roy, "Soft set theory," Computers \& Mathematics with Applications, vol. 45, no. 4-5, pp. 555-562, 2003.

[20] P. K. Maji, A. R. Roy, and R. Biswas, "An application of soft sets in A decision making problem," Computers \& Mathematics with Applications, vol. 44, no. 8-9, pp. 1077-1083, 2002.

[21] P. K. Maji, R. Biswas, and A. R. Roy, "Fuzzy soft sets," Journal of Fuzzy Mathematics, vol. 9, pp. 589-602, 2001.

[22] P. K. Maji, R. Biswas, and A. Roy, "Intuitionistic fuzzy soft sets," Journal of Fuzzy Mathematics, vol. 9, pp. 677-692, 2001.

[23] H. Garg and R. Arora, "Generalized and group-based generalized intuitionistic fuzzy soft sets with applications in decision-making," Applied Intelligence, vol. 48, no. 2, pp. 343-356, 2018.

[24] H. Garg, "New ranking method for normal intuitionistic sets under crisp, interval environments and its applications to multiple attribute decision making process," Complex \& Intelligent Systems, vol. 6, no. 3, pp. 559-571, 2020.

[25] T. Mahmood and Z. Ali, "Entropy measure and TOPSIS method based on correlation coefficient using complex q-rung orthopair fuzzy information and its application to multi-attribute decision making," Soft Computing, vol. 11, pp. 1-27, 2020.

[26] H. Garg and R. Arora, "Maclaurin symmetric mean aggregation operators based on t-norm operations for the dual hesitant fuzzy soft set," Journal of Ambient Intelligence and Humanized Computing, vol. 11, no. 1, pp. 375-410, 2020.

[27] R. Arora and H. Garg, "A robust correlation coefficient measure of dual hesitant fuzzy soft sets and their application in decision making," Engineering Applications of Artificial Intelligence, vol. 72, pp. 80-92, 2018.

[28] R. Arora and H. Garg, "Group decision-making method based on prioritized linguistic intuitionistic fuzzy aggregation operators and its fundamental properties," Journal of Computational and Applied Mathematics, vol. 38, no. 2, pp. 1-36, 2019.

[29] H. Garg and R. Arora, "Generalized maclaurin symmetric mean aggregation operators based on Archimedean t-norm of the intuitionistic fuzzy soft set information," Artifcial Intelligence Review, pp. 1-41, Springer Verlag, Berlin, Germany, 2020.

[30] H. Garg and R. AroraArora, "TOPSIS method based on correlation coefficient for solving decision-making problems with intuitionistic fuzzy soft set information," AIMS Mathematics, vol. 5, no. 4, pp. 2944-2966, 2020.

[31] X. Peng, Y. Yang, and J. Song, "Pythagorean fuzzy soft set and its application," Computer Engineering, vol. 41, pp. 224-229, 2015.

[32] T. M. Athira, S. J. John, and H. Garg, "A novel entropy measure of Pythagorean fuzzy soft sets," AIMS Mathematics, vol. 5, no. 2, pp. 1050-1061, 2020.

[33] T. M. Athira, S. J. John, and H. Garg, "Entropy and distance measures of Pythagorean fuzzy soft sets and their applications," Journal of Intelligent \& Fuzzy Systems, vol. 37, no. 3, pp. 4071-4084, 2019.

[34] K. Naeem, M. Riaz, X. Peng, and D. Afzal, "Pythagorean fuzzy soft MCGDM methods based on TOPSIS, VIKOR and 
aggregation operators," Journal of Intelligent \& Fuzzy Systems, vol. 37, no. 5, pp. 6937-6957, 2019.

[35] M. Riaz, K. Naeem, and D. Afzal, "Pythagorean m-polar fuzzy soft sets with TOPSIS method for MCGDM," Punjab University Journal of Mathematics, vol. 52, no. 3, pp. 21-46, 2020a.

[36] M. Riaz, N. Khalid, and D. Afzal, "A similarity measure under pythagorean fuzzy soft environment with applications," Computational and Applied Mathematics, vol. 39, no. 4, pp. 1-17, 2020b.

[37] C. L. Hwang and K. Yoon, Multiple Attribute Decision Making Methods and Applications A State-Of-The-Art Survey, Springer-Verlag Berlin Heidelberg, Berlin, Germany, 1981.

[38] M. Zulqarnain, F. Dayan, and M. Saeed, “TOPSIS analysis for the prediction of diabetes based on general characteristics of humans," International Journal of Pharmaceutical Sciences and Research, vol. 9, no. 7, pp. 2932-2939, 2018.

[39] A. Sarkar, "A TOPSIS method to evaluate the technologies," International Journal of Quality \& Reliability Management, vol. 31, no. 1, pp. 2-13, 2013.

[40] R. M. Zulqarnain, S. Abdal, A. Maalik et al., "Application of TOPSIS method in decision making via soft set," Biomedical Journal of Scientific \& Technical Research, vol. 24, no. 3, pp. 18208-18215, 2020.

[41] R. M. Zulqarnain, S. Abdal, B. Ali et al., "Selection of medical clinic for disease diagnosis by using TOPSIS method," International Journal of Pharmaceutical Sciences Review and Research, vol. 61, no. 1, pp. 22-27, 2020.

[42] C.-T. Chen, "Extensions of the TOPSIS for group decisionmaking under fuzzy environment," Fuzzy Sets and Systems, vol. 114, no. 1, pp. 1-9, 2000.

[43] R. M. Zulqarnain, X. L. Xin, M. Saeed, N. Ahmad, F. Dayan, and B. Ahmad, "Recruitment of medical staff in health department by using TOPSIS method," International Journal of Pharmaceutical Sciences Review and Research, vol. 62, no. 1, pp. 1-7, 2020.

[44] R. M. Zulqarnain, M. Saeed, N. Ahmad, F. Dayan, and B. Ahmad, "Application of TOPSIS method for decision making," International Journal of Scientific Research in Mathematical and Statistical Sciences, vol. 7, no. 2, pp. 76-81, 2020.

[45] L. Dymova, P. Sevastjanov, and A. Tikhonenko, "An approach to generalization of fuzzy TOPSIS method," Information Sciences, vol. 238, pp. 149-162, 2013.

[46] M. Zulqarnain and F. Dayan, "Choose best criteria for decision making via fuzzy topsis method," Mathematics and Computer Science, vol. 2, no. 6, pp. 113-119, 2017.

[47] R. M. Zulqarnain, M. Saeed, B. Ali et al., "Generalized fuzzy TOPSIS to solve multi-criteria decision-making problems," Journal of New Theory, vol. 32, pp. 40-50, 2020.

[48] A. Y. Yayla, A. Yildiz, and O. Ahmet, "Fuzzy TOPSIS method in supplier selection and application in the garment industry," Fibres and Textiles in Eastern Europe, vol. 93, no. 4, pp. 20-23, 2012.

[49] R. M. Zulqarnain, X. L. Xin, M. Saeed, F. Smarandache, and N. Ahmad, "Generalized neutrosophic TOPSIS to solve multicriteria decision-making problems," Neutrosophic Sets and Systems, vol. 38, pp. 276-292, 2020.

[50] A. Biswas and S. Kumar, An Integrated TOPSIS Approach to MADM with Interval-Valued Intuitionistic Fuzzy Settings, Advanced Computational and Communication Paradigms, Springer, Berlin, Germany, 2018.

[51] M. Zulqarnain and F. Dayan, "Selection of best alternative for an automotive company by intuitionistic fuzzy TOPSIS method," International Journal of Scientific \& Technology Research, vol. 6, no. 10, pp. 126-132, 2017.

[52] F. Smarandache, "Extension of soft set to hypersoft set, and then to plithogenic hypersoft set," Neutrosophic Sets and Systems, vol. 22, pp. 168-170, 2018.

[53] S. Rana, M. Qayyum, M. Saeed, and F. Smarandache, "Plithogenic fuzzy whole hypersoft set: construction of operators and their application in frequency matrix multi attribute decision making Technique," Neutrosophic Sets and Systems, vol. 28, pp. 34-50, 2019.

[54] R. M. Zulqarnain, X. L. Xin, M. Saqlain, and F. Smarandache, "Generalized aggregate operators on neutrosophic hypersoft set," Neutrosophic Sets and Systems, vol. 36, pp. 271-281, 2020.

[55] S. Alkhazaleh, "Plithogenic Soft Set," Neutrosophic Sets and Systems, vol. 33, pp. 256-274, 2020.

[56] R. M. Zulqarnain, X. L. Xin, and M. Saeed, "Extension of TOPSIS method under intuitionistic fuzzy hypersoft environment based on correlation coefficient and aggregation operators to solve decision making problem," AIMS Mathematics, vol. 6, no. 3, pp. 2732-2755, 2020.

[57] Z. Yang, X. Li, H. Garg, and M. Qi, "Decision support algorithm for selecting an antivirus mask over COVID-19 pandemic under spherical normal fuzzy environment," International Journal of Environmental Research and Public Health, vol. 17, no. 10, p. 3407, 2020.

[58] G. Shahzadi and M. Akram, "Group decision-making for the selection of an antivirus mask under fermatean fuzzy soft information," Journal of Intelligent \& Fuzzy Systems, vol. 40, no. 1, p. 1401, 2021.

[59] R. Arora and H. Garg, "Robust aggregation operators for multi-criteria decision-making with intuitionistic fuzzy soft set environment," Scientia Iranica, vol. 25, no. 2, pp. 931-942, 2018.

[60] A. Raza, M. Farman, A. Akgül, M. S. Iqbal, and A. Ahmad, "Simulation and numerical solution of fractional order Ebola virus model with novel technique," AIMS Bioengineering, vol. 7, no. 4, pp. 194-207, 2020.

[61] A. Ahmed, B. Salam, B. Salam, M. Mohammad, A. AkgülH, and S. Khoshnaw, "Analysis coronavirus disease (COVID-19) model using numerical approaches and logistic model," Aims Bioengineering, vol. 7, no. 3, pp. 130-146, 2020.

[62] H. M. Zhang, Z. S. Xu, and Q. Chen, "On clustering approach to intuitionistic fuzzy sets," Control Decision, vol. 22, pp. 882-888, 2007. 